Special Issue: 25 years of the Mizar Mathematical Library

FORMALIZED MATHEMATICS

Vol. 22, No. 2, Pages $125-155,2014$

DOI: 10.2478 /forma-2014-0015

\title{
Term Context
}

\author{
Grzegorz Bancerek \\ Association of Mizar Users \\ Białystok, Poland
}

\begin{abstract}
Summary. Two construction functors: simple term with a variable and compound term with an operation and argument terms and schemes of term induction are introduced. The degree of construction as a number of used operation symbols is defined. Next, the term context is investigated. An $x$-context is a term which includes a variable $x$ once only. The compound term is $x$-context iff the argument terms include an $x$-context once only. The context induction is shown and used many times. As a key concept, the context substitution is introduced. Finally, the translations and endomorphisms are expressed by context substitution.
\end{abstract}

MSC: 08A35 03B35

Keywords: construction degree; context; translation; endomorphism

MML identifier: MSAFREE5, version: 8.1.03 5.23.1210

The notation and terminology used in this paper have been introduced in the following articles: [1, [2], 3], 4], [6], [43], [24], [22], [26], [53], [33], [45], [27], [28], 29], 8], 25], 9], [51], 39], [46], [47], [41], [48], 223, [10], [1], 49], [36], [37], [12, [13], 14], 15], [31, [50], [34, [55], [56, 16], 38, [54, 11], 18], 19], [20], 21], 35], and 32].

\section{Preliminaries}

Let $\Sigma$ be a non empty non void many sorted signature, $\mathfrak{A}$ be a non-empty algebra over $\Sigma$, and $\sigma$ be a sort symbol of $\Sigma$.

An element of $\mathfrak{A}$ from $\sigma$ is an element of (the sorts of $\mathfrak{A})(\sigma)$. From now on $a, b$ denote objects, $I, J$ denote sets, $f$ denotes a function, $R$ denotes a binary relation, $i, j, n$ denote natural numbers, $m$ denotes an element of $\mathbb{N}, \Sigma$ denotes a non empty non void many sorted signature, $\sigma, \sigma_{1}, \sigma_{2}$ denote sort symbols of $\Sigma, o$ denotes an operation symbol of $\Sigma, X$ denotes a non-empty many sorted set 
indexed by the carrier of $\Sigma, x, x_{1}, x_{2}$ denote elements of $X(\sigma), x_{11}$ denotes an element of $X\left(\sigma_{1}\right), T$ denotes a free in itself including $\Sigma$-terms over $X$ algebra over $\Sigma$ with all variables and inheriting operations, $g$ denotes a translation in $\mathfrak{F}_{\Sigma}(X)$ from $\sigma_{1}$ into $\sigma_{2}$, and $h$ denotes an endomorphism of $\mathfrak{F}_{\Sigma}(X)$.

Let us consider $\Sigma$ and $X$. Let $T$ be an including $\Sigma$-terms over $X$ algebra over $\Sigma$ with all variables and $\rho$ be an element of $T$. The functor ${ }^{\circledR} \rho$ yielding an element of $\mathfrak{F}_{\Sigma}(X)$ is defined by the term

(Def. 1) $\rho$.

Let us consider $T$. Observe that every element of $T$ is finite and every set which is natural-membered is also $\subseteq$-linear.

In the sequel $\rho, \rho_{1}, \rho_{2}$ denote elements of $T$ and $\tau, \tau_{1}, \tau_{2}$ denote elements of $\mathfrak{F}_{\Sigma}(X)$.

Let us consider $\Sigma$. Let $\mathfrak{A}$ be an algebra over $\Sigma$. Let us consider $a$. We say that $a \in \mathfrak{A}$ if and only if

(Def. 2) $\quad a \in \bigcup($ the sorts of $\mathfrak{A})$.

Let us consider $b$. We say that $b$ is $a$-different if and only if

(Def. 3) $b \neq a$.

Let $I$ be a non trivial set. Note that there exists an element of $I$ which is $a$-different.

Now we state the proposition:

(1) Let us consider trees $\tau, \tau_{1}$ and finite sequences $p, q$ of elements of $\mathbb{N}$. Suppose

(i) $p \in \tau$, and

(ii) $q \in \tau$ with-replacement $\left(p, \tau_{1}\right)$.

Then

(iii) if $p \npreceq q$, then $q \in \tau$, and

(iv) for every finite sequence $\rho$ of elements of $\mathbb{N}$ such that $q=p^{\frown} \rho$ holds $\rho \in \tau_{1}$.

Proof: If $p \npreceq q$, then $q \in \tau$ by [17, (1)].

Let $R$ be a finite binary relation. Let us consider $a$. Let us note that $\operatorname{Coim}(R, a)$ is finite.

Let us consider finite sequences $p, q, \rho$. Now we state the propositions:

(2) If $p^{\frown} q \preceq \rho$, then $p \preceq \rho$.

(3) If $p \frown q \preceq p \frown \rho$, then $q \preceq \rho$.

Now we state the propositions:

(4) Let us consider finite sequences $p, q$. Suppose $i \leqslant \operatorname{len} p$. Then $\left(p^{\frown}\right.$ q) $\uparrow \operatorname{Seg} i=p \nmid \operatorname{Seg} i$.

(5) Let us consider finite sequences $p, q$, $\rho$. If $q \preceq p^{\frown} \rho$, then $q \preceq p$ or $p \preceq q$. The theorem is a consequence of (4). 
Let us consider $\Sigma$. We say that $\Sigma$ is sufficiently rich if and only if

(Def. 4) There exists $o$ such that $\sigma \in \operatorname{rng} \operatorname{Arity}(o)$.

We say that $\Sigma$ is growable if and only if

(Def. 5) There exists $\tau$ such that height $\operatorname{dom} \tau=n$.

Let us consider $n$. We say that $\Sigma$ is $n$-ary operation including if and only if

(Def. 6) There exists $o$ such that len $\operatorname{Arity}(o)=n$.

Let us note that there exists a non empty non void many sorted signature which is $n$-ary operation including and there exists a non empty non void many sorted signature which is sufficiently rich.

Let us consider $R$. We say that $R$ is nontrivial if and only if

(Def. 7) If $I \in \operatorname{rng} R$, then $I$ is not trivial.

We say that $R$ is infinite-yielding if and only if

(Def. 8) If $I \in \operatorname{rng} R$, then $I$ is infinite.

Let us observe that every binary relation which is nontrivial is also nonempty and every binary relation which is infinite-yielding is also nontrivial.

Let $I$ be a set. Observe that there exists a many sorted set indexed by $I$ which is infinite-yielding and there exists a finite sequence which is infinite-yielding.

Let $I$ be a non empty set, $f$ be a nontrivial many sorted set indexed by $I$, and $a$ be an element of $I$. Let us note that $f(a)$ is non trivial.

Let $f$ be an infinite-yielding many sorted set indexed by $I$. Note that $f(a)$ is infinite.

Let us consider $\Sigma, X$, and $o$. Let us note that every element of $\operatorname{Args}\left(o, \mathfrak{F}_{\Sigma}(X)\right)$ is decorated tree yielding.

In the sequel $Y$ denotes an infinite-yielding many sorted set indexed by the carrier of $\Sigma, y, y_{1}$ denote elements of $Y(\sigma), y_{11}$ denotes an element of $Y\left(\sigma_{1}\right)$, $Q$ denotes a free in itself including $\Sigma$-terms over $Y$ algebra over $\Sigma$ with all variables and inheriting operations, $q, q_{1}$ denote elements of $\operatorname{Args}\left(o, \mathfrak{F}_{\Sigma}(Y)\right), u$, $u 1, u 2$ denote elements of $Q, v, v_{1}, v_{2}$ denote elements of $\mathfrak{F}_{\Sigma}(Y), Z$ denotes a nontrivial many sorted set indexed by the carrier of $\Sigma, z, z_{1}$ denote elements of $Z(\sigma), l, l 1$ denote elements of $\mathfrak{F}_{\Sigma}(Z), R$ denotes a free in itself including $\Sigma$-terms over $Z$ algebra over $\Sigma$ with all variables and inheriting operations, and $k, k 1$ denote elements of $\operatorname{Args}\left(o, \mathfrak{F}_{\Sigma}(Z)\right)$.

Let $p$ be a finite sequence. Note that $p \frown \emptyset$ reduces to $p$ and $\emptyset \frown p$ reduces to $p$.

Let $I$ be a finite sequence-membered set. The functor $p \frown I$ yielding a set is defined by the term

(Def. 9) $\quad\left\{p^{\frown} q\right.$, where $q$ is an element of $\left.I: q \in I\right\}$.

Let us observe that $p \frown I$ is finite sequence-membered.

Let $f$ be a finite sequence and $E$ be an empty set. One can verify that $f \frown E$ reduces to $E$. 
Let $p$ be a decorated tree yielding finite sequence. Let us consider $a$. Let us note that $p(a)$ is relation-like and every set which is tree-like is also finite sequence-membered.

Let $p$ be a decorated tree yielding finite sequence. Let us consider $a$. One can check that $\operatorname{dom}(p(a))$ is finite sequence-membered.

Let $\tau, \tau_{1}$ be trees. One can check that $\tau_{1}$ with-replacement $\left(\varepsilon_{\mathbb{N}}, \tau\right)$ reduces to $\tau$.

Let $d, d_{1}$ be decorated trees. One can check that $d_{1}$ with-replacement $\left(\varepsilon_{\mathbb{N}}, d\right)$ reduces to $d$.

Now we state the proposition:

(6) Let us consider finite sequences $\xi, w$ of elements of $\mathbb{N}$, tree yielding finite sequences $p, q$, and trees $d, \tau$. Suppose

(i) $i<\operatorname{len} p$, and

(ii) $\xi=\langle i\rangle\urcorner w$, and

(iii) $d=p(i+1)$, and

(iv) $q=p+\cdot(i+1, d$ with-replacement $(w, \tau))$, and

(v) $\xi \in \overbrace{p}$.

Then $\overbrace{p}$ with-replacement $(\xi, \tau)=\overbrace{q}$. The theorem is a consequence of (2).

Let $F$ be a function yielding function and $f$ be a function. Let us consider $a$. Note that $F+\cdot(a, f)$ is function yielding.

Now we state the propositions:

(7) Let us consider a function yielding function $F$ and a function $f$. Then $\operatorname{dom}_{\kappa}(F+\cdot(a, f))(\kappa)=\operatorname{dom}_{\kappa} F(\kappa)+\cdot(a, \operatorname{dom} f)$.

(8) Let us consider finite sequences $\xi, w$ of elements of $\mathbb{N}$, decorated tree yielding finite sequences $p, q$, and decorated trees $d, \tau$. Suppose

(i) $i<\operatorname{len} p$, and

(ii) $\xi=\langle i\rangle\urcorner w$, and

(iii) $d=p(i+1)$, and

(iv) $q=p+\cdot(i+1, d$ with-replacement $(w, \tau))$, and

(v) $\xi \in \overbrace{\operatorname{dom} p(\kappa)}$.

Then $(a$-tree $(p))$ with-replacement $(\xi, \tau)=a$-tree $(q)$. The theorem is a consequence of $(7),(6),(2)$, and (3).

(9) Let us consider a set $a$ and a decorated tree yielding finite sequence $w$. Then $\operatorname{dom}(a$-tree $(w))=\{\emptyset\} \cup \bigcup\{\langle i\rangle \frown \operatorname{dom}(w(i+1)): i<\operatorname{len} w\}$. Proof: Set $\mathfrak{A}=\{\langle i\rangle \frown \operatorname{dom}(w(i+1)): i<\operatorname{len} w\} \cdot \operatorname{dom}(a$-tree $(w)) \subseteq\{\emptyset\} \cup \cup \mathfrak{A}$ by [20, (11)]. 
Let $p$ be a decorated tree yielding finite sequence. Let us consider $a$ and $I$. Note that $p(a)^{-1}(I)$ is finite sequence-membered.

Now we state the proposition:

(10) Let us consider a finite sequence-membered set $I$ and a finite sequence $p$. Then $\overline{\overline{p^{-I}}}=\overline{\bar{I}}$. Proof: Define $\mathcal{F}$ (element of $\left.I\right)=p^{\frown} \$_{1}$. Consider $f$ such that $\operatorname{dom} f=I$ and for every element $q$ of $I$ such that $q \in I$ holds $f(q)=\mathcal{F}(q)$ from [7, Sch. 2]. $\operatorname{rng} f=p^{\frown} I . f$ is one-to-one by [22, (33)].

Let $I$ be a finite finite sequence-membered set and $p$ be a finite sequence. Note that $p^{\frown I} I$ is finite.

Now we state the proposition:

(11) Let us consider finite sequence-membered sets $I, J$ and finite sequences $p, q$. Suppose

(i) len $p=$ len $q$, and

(ii) $p \neq q$.

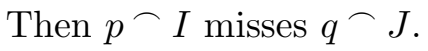

Let us consider $i$. Let us note that $\overline{\bar{i}}$ reduces to $i$. Let us consider $j$. We identify $i+j$ with $i+j$.

The scheme CardUnion deals with a unary functor $\mathcal{I}$ yielding a set and a finite sequence $f$ of elements of $\mathbb{N}$ and states that

(Sch. 1) $\overline{\overline{\bigcup U \mathcal{I}(i): i<\operatorname{len} f\}}}=\sum f$

provided

- for every $i$ and $j$ such that $i<\operatorname{len} f$ and $j<\operatorname{len} f$ and $i \neq j$ holds $\mathcal{I}(i)$ misses $\mathcal{I}(j)$ and

- for every $i$ such that $i<\operatorname{len} f$ holds $\overline{\overline{\mathcal{I}(i)}}=f(i+1)$.

Let $f$ be a finite sequence. Note that $\{f\}$ is finite sequence-membered.

Now we state the propositions:

(12) Let us consider finite sequences $f, g$. Then $f \frown\{g\}=\left\{f^{\frown} g\right\}$.

(13) Let us consider finite sequence-membered sets $I, J$ and a finite sequence $f$. Then $I \subseteq J$ if and only if $f \frown I \subseteq f \frown J$.

In the sequel $c, c_{1}, c_{2}$ denote sets and $d, d_{1}$ denote decorated trees.

Now we state the proposition:

(14) Leaves(the elementary tree of 0$)=\{\emptyset\}$.

Let us note that sethood property holds for trees.

Now we state the propositions:

(15) Let us consider a non empty tree yielding finite sequence $p$.

Then Leaves $(\overbrace{p})=\{\langle i\rangle \frown q$, where $q$ is a finite sequence of elements of $\mathbb{N}, d$ is a tree : $q \in \operatorname{Leaves}(d)$ and $i+1 \in \operatorname{dom} p$ and $d=p(i+1)\}$. 
Proof: Set $i_{0}=$ the element of $\operatorname{dom} p$. Leaves $(\overbrace{p}) \subseteq\{\langle i\rangle\urcorner q$, where $q$ is a finite sequence of elements of $\mathbb{N}, d$ is a tree : $q \in \operatorname{Leaves}(d)$ and $i+1 \in \operatorname{dom} p$ and $d=p(i+1)\}$ by [13, (11), (13)], [52, (25)], [17, (1)].

(16) Leaves(the root tree of $c)=\{c\}$.

(17) $\operatorname{dom} d \subseteq \operatorname{dom} d_{c \leftarrow d_{1}}$.

Let us consider $c$ and $d$. Observe that (the root tree of $c)_{c \leftarrow d}$ reduces to $d$. Now we state the proposition:

(18) Suppose $c_{1} \neq c_{2}$. Then (the root tree of $\left.c_{1}\right)_{c_{2} \leftarrow d}=$ the root tree of $c_{1}$. PROOF: $\operatorname{dom}\left(\text { the root tree of } c_{1}\right)_{c_{2} \leftarrow d}=\operatorname{dom}\left(\right.$ the root tree of $c_{1}$ ) by [20, (3)], [17, (29)], [40, (15)].

Let $f$ be a non empty function yielding function. Note that $\operatorname{dom}_{\kappa} f(\kappa)$ is non empty and $\operatorname{rng}_{\kappa} f(\kappa)$ is non empty.

Now we state the proposition:

(19) Let us consider non empty decorated tree yielding finite sequences $p, q$. Suppose

(i) $\operatorname{dom} q=\operatorname{dom} p$, and

(ii) for every $i$ and $d_{1}$ such that $i \in \operatorname{dom} p$ and $d_{1}=p(i)$ holds $q(i)=$ $d_{1 c \leftarrow d}$.

Then $(b \text {-tree }(p))_{c \leftarrow d}=b$-tree $(q)$. Proof: Leaves $(\overbrace{\operatorname{dom}_{\kappa} p(\kappa)})=\{\langle i\rangle\urcorner q$, where $q$ is a finite sequence of elements of $\mathbb{N}, d$ is a tree : $q \in \operatorname{Leaves}(d)$ and $i+1 \in \operatorname{dom}\left(\operatorname{dom}_{\kappa} p(\kappa)\right)$ and $\left.d=\left(\operatorname{dom}_{\kappa} p(\kappa)\right)(i+1)\right\} \cdot \operatorname{dom}(b \text {-tree }(p))_{c \leftarrow d}=$ $\operatorname{dom}(b$-tree $(q))$ by [17, (22)], [13, (11), (13)], [52, (25)].

Let us consider $\Sigma$ and $\sigma$. Let $\mathfrak{A}$ be a non empty algebra over $\Sigma$ and $a$ be an element of $\mathfrak{A}$. We say that $a$ is $\sigma$-sort if and only if

(Def. 10) $a \in($ the sorts of $\mathfrak{A})(\sigma)$.

Let $\mathfrak{A}$ be a non-empty algebra over $\Sigma$. One can verify that there exists an element of $\mathfrak{A}$ which is $\sigma$-sort and every element of (the sorts of $\mathfrak{A})(\sigma)$ is $\sigma$-sort.

Let $\mathfrak{A}$ be a non empty algebra over $\Sigma$. Assume $\mathfrak{A}$ is disjoint valued. Let $a$ be an element of $\mathfrak{A}$. The functor the sort of $a$ yielding a sort symbol of $\Sigma$ is defined by

(Def. 11) $\quad a \in($ the sorts of $\mathfrak{A})(i t)$.

Now we state the propositions:

(20) Let us consider a disjoint valued non-empty algebra $\mathfrak{A}$ over $\Sigma$ and a $\sigma$-sort element $a$ of $\mathfrak{A}$. Then the sort of $a=\sigma$.

(21) Let us consider a disjoint valued non empty algebra $\mathfrak{A}$ over $\Sigma$. Then every element of $\mathfrak{A}$ is (the sort of $a$ )-sort.

(22) The sort of ${ }^{\circledR} \rho=$ the sort of $\rho$. 
(23) Let us consider an element $\rho$ of (the sorts of $T)(\sigma)$. Then the sort of $\rho=\sigma$.

(24) Let us consider a term $u$ of $\Sigma$ over $X$. Suppose $\tau=u$. Then the sort of $\tau=$ the sort of $u$.

Let us consider $\Sigma, X, o$, and $T$. One can verify that every element of $\operatorname{Args}(o, T)$ is $(\bigcup($ the sorts of $T))$-valued.

Now we state the proposition:

(25) Let us consider an element $q$ of $\operatorname{Args}(o, T)$. Suppose $i \in \operatorname{dom} q$. Then the sort of $q_{i}=\operatorname{Arity}(o)_{i}$.

Let us consider $\Sigma$. Let $\mathfrak{A}, \mathcal{B}$ be non-empty algebras over $\Sigma$ and $f$ be a many sorted function from $\mathfrak{A}$ into $\mathcal{B}$. Assume $\mathfrak{A}$ is disjoint valued. Let $a$ be an element of $\mathfrak{A}$. The functor $f(a)$ yielding an element of $\mathcal{B}$ is defined by the term

(Def. 12) $f($ the sort of $a)(a)$.

Let us consider a disjoint valued non-empty algebra $\mathfrak{A}$ over $\Sigma$, a non-empty algebra $\mathcal{B}$ over $\Sigma$, a many sorted function $f$ from $\mathfrak{A}$ into $\mathcal{B}$, and an element $a$ of (the sorts of $\mathfrak{A})(\sigma)$. Now we state the propositions:

(26) $f(a)=f(\sigma)(a)$.

(27) $f(a)$ is an element of (the sorts of $\mathcal{B})(\sigma)$. The theorem is a consequence of (26).

Now we state the propositions:

(28) Let us consider disjoint valued non-empty algebras $\mathfrak{A}, \mathcal{B}$ over $\Sigma$, a many sorted function $f$ from $\mathfrak{A}$ into $\mathcal{B}$, and an element $a$ of $\mathfrak{A}$. Then the sort of $f(a)=$ the sort of $a$.

(29) Let us consider disjoint valued non-empty algebras $\mathfrak{A}, \mathcal{B}$ over $\Sigma$, a nonempty algebra $\mathcal{C}$ over $\Sigma$, a many sorted function $f$ from $\mathfrak{A}$ into $\mathcal{B}$, a many sorted function $g$ from $\mathcal{B}$ into $\mathcal{C}$, and an element $a$ of $\mathfrak{A}$. Then $(g \circ f)(a)=$ $g(f(a))$. The theorem is a consequence of (28).

(30) Let us consider a disjoint valued non-empty algebra $\mathfrak{A}$ over $\Sigma$, a nonempty algebra $\mathcal{B}$ over $\Sigma$, and many sorted functions $f_{1}, f_{2}$ from $\mathfrak{A}$ into $\mathcal{B}$. If for every element $a$ of $\mathfrak{A}, f_{1}(a)=f_{2}(a)$, then $f_{1}=f_{2}$. The theorem is a consequence of (26).

Let us consider $\Sigma$. Let $\mathfrak{A}, \mathcal{B}$ be algebras over $\Sigma$. Assume there exists a many sorted function $h$ from $\mathfrak{A}$ into $\mathcal{B}$ such that $h$ is a homomorphism of $\mathfrak{A}$ into $\mathcal{B}$.

A homomorphism from $\mathfrak{A}$ to $\mathcal{B}$ is a many sorted function from $\mathfrak{A}$ into $\mathcal{B}$ and is defined by

(Def. 13) it is a homomorphism of $\mathfrak{A}$ into $\mathcal{B}$.

Now we state the proposition:

(31) Let us consider a many sorted function $h$ from $\mathfrak{F}_{\Sigma}(X)$ into $T$. Then $h$ is a homomorphism from $\mathfrak{F}_{\Sigma}(X)$ to $T$ if and only if $h$ is a homomorphism of 
$\mathfrak{F}_{\Sigma}(X)$ into $T$.

Let us consider $\Sigma, X$, and $T$. Observe that the functor the canonical homomorphism of $T$ yields a homomorphism from $\mathfrak{F}_{\Sigma}(X)$ to $T$. Let us consider $\rho$. One can check that (the canonical homomorphism of $T)\left({ }^{@} \rho\right)$ reduces to $\rho$.

Now we state the proposition:

(32) Suppose $\tau_{2}=$ (the canonical homomorphism of $\left.T\right)\left(\tau_{1}\right)$.

Then (the canonical homomorphism of $T)\left(\tau_{1}\right)=$ (the canonical homomorphism of $T)\left(\tau_{2}\right)$. The theorem is a consequence of (22) and (28).

\section{Constructing Terms}

In the sequel $w$ denotes an element of $\operatorname{Args}(o, T)$ and $p, p_{1}$ denote elements of $\operatorname{Args}\left(o, \mathfrak{F}_{\Sigma}(X)\right)$.

Let us consider $\Sigma, X, \sigma$, and $x$. The functor $x$-term yielding an element of (the sorts of $\left.\mathfrak{F}_{\Sigma}(X)\right)(\sigma)$ is defined by the term

(Def. 14) The root tree of $\langle x, \sigma\rangle$.

Let us consider $o$ and $p$. The functor $o$-term $p$ yielding an element of $\mathfrak{F}_{\Sigma}(X)$ from the result sort of $o$ is defined by the term

(Def. 15) $\langle o$, the carrier of $\Sigma\rangle$-tree $(p)$.

Now we state the propositions:

(33) The sort of $x$-term $=\sigma$.

(34) The sort of $o$-term $p=$ the result sort of $o$. The theorem is a consequence of (24).

(35) Let us consider an object $i$. Then $i \in($ FreeGenerator $(T))(\sigma)$ if and only if there exists $x$ such that $i=x$-term.

Let us consider $\Sigma, X, \sigma$, and $x$. Let us note that $x$-term is non compound.

Let us consider $o$ and $p$. One can check that $o$-term $p$ is compound and (the result sort of $o$ )-sort.

Now we state the propositions:

(36) (i) there exists $\sigma$ and there exists $x$ such that $\tau=x$-term, or

(ii) there exists $o$ and there exists $p$ such that $\tau=o$-term $p$.

(37) If $\tau$ is not compound, then there exists $\sigma$ and there exists $x$ such that $\tau=x$-term.

(38) If $\tau$ is compound, then there exists $o$ and there exists $p$ such that $\tau=$ $o$-term $p$.

(39) $x$-term $\neq o$-term $p$.

Let us consider $\Sigma$. Let $X$ be a non-empty many sorted set indexed by the carrier of $\Sigma$. Note that there exists an element of $\mathfrak{F}_{\Sigma}(X)$ which is compound. 
Let us consider $X$. Let $e$ be a compound element of $\mathfrak{F}_{\Sigma}(X)$. Let us note that the functor main-constr $e$ yields an operation symbol of $\Sigma$. One can check that the functor $\operatorname{args} e$ yields an element of $\operatorname{Args}\left(\right.$ main-constr $\left.e, \mathfrak{F}_{\Sigma}(X)\right)$. Now we state the propositions:

(40) $\operatorname{args}(x$-term $)=\emptyset$.

(41) Let us consider a compound element $\tau$ of $\mathfrak{F}_{\Sigma}(X)$.

Then $\tau=$ main-constr $\tau$-term $\operatorname{args} \tau$. The theorem is a consequence of (38).

(42) $x$-term $\in T$.

Let us consider $\Sigma, X, T, \sigma$, and $x$. Note that (the canonical homomorphism of $T)(x$-term $)$ reduces to $x$-term.

The scheme TermInd deals with a unary predicate $\mathcal{P}$ and a non empty non void many sorted signature $\Sigma$ and a non-empty many sorted set $\mathcal{X}$ indexed by the carrier of $\Sigma$ and an element $\tau$ of $\mathfrak{F}_{\Sigma}(\mathcal{X})$ and states that

(Sch. 2) $\mathcal{P}[\tau]$

provided

- for every sort symbol $\sigma$ of $\Sigma$ and for every element $x$ of $\mathcal{X}(\sigma), \mathcal{P}[x$-term $]$ and

- for every operation symbol $o$ of $\Sigma$ and for every element $p$ of $\operatorname{Args}\left(o, \mathfrak{F}_{\Sigma}(\mathcal{X})\right)$ such that for every element $\tau$ of $\mathfrak{F}_{\Sigma}(\mathcal{X})$ such that $\tau \in \operatorname{rng} p$ holds $\mathcal{P}[\tau]$ holds $\mathcal{P}[o-\operatorname{term} p]$.

The scheme TermAlgebraInd deals with a unary predicate $\mathcal{P}$ and a non empty non void many sorted signature $\Sigma$ and a non-empty many sorted set $\mathcal{X}$ indexed by the carrier of $\Sigma$ and a free in itself including $\Sigma$-terms over $\mathcal{X}$ algebra $\mathfrak{A}$ over $\Sigma$ with all variables and inheriting operations and an element $\tau$ of $\mathfrak{A}$ and states that

$($ Sch. 3) $\mathcal{P}[\tau]$

provided

- for every sort symbol $\sigma$ of $\Sigma$ and for every element $x$ of $\mathcal{X}(\sigma)$ and for every element $\rho$ of $\mathfrak{A}$ such that $\rho=x$-term holds $\mathcal{P}[\rho]$ and

- for every operation symbol $o$ of $\Sigma$ and for every element $p$ of $\operatorname{Args}\left(o, \mathfrak{F}_{\Sigma}(\mathcal{X})\right)$ and for every element $\rho$ of $\mathfrak{A}$ such that $\rho=o$-term $p$ and for every element $\tau$ of $\mathfrak{A}$ such that $\tau \in \operatorname{rng} p$ holds $\mathcal{P}[\tau]$ holds $\mathcal{P}[\rho]$. 


\section{Construction Degree}

Let us consider $\Sigma, X, T$, and $\rho$. The functors: the construction degree of $\rho$ and height $\rho$ yielding natural numbers are defined by terms,

(Def. 16) $\overline{\overline{\rho^{-1}(\alpha \times\{\beta\})}}$, where $\alpha$ is the carrier' of $\Sigma$ and $\beta$ is the carrier of $\Sigma$,

(Def. 17) height dom $\rho$,

respectively. We introduce $\operatorname{deg} \rho$ as a synonym of the construction degree of $\rho$.

Now we state the propositions:

(43) $\operatorname{deg}{ }^{\circledR} \rho=\operatorname{deg} \rho$.

(44) height ${ }^{@} \rho=$ height $\rho$.

(45) $\operatorname{height}(x$-term $)=0$.

One can verify that every set which is natural-membered is also ordinalmembered and finite-membered.

Let $I$ be a finite natural-membered set. One can verify that $\bigcup I$ is natural.

Let $I$ be a non empty finite natural-membered set. We identify $U I$ with $\max I$. Now we state the propositions:

(i) $\left\{\right.$ height $\left.\tau_{1}: \tau_{1} \in \operatorname{rng} p\right\}$ is natural-membered and finite, and

(ii) $\bigcup\{$ height $\tau: \tau \in \operatorname{rng} p\}$ is a natural number.

Proof: Set $I=\{$ height $\tau: \tau \in \operatorname{rng} p\}$. $I$ is natural-membered. Define $\mathcal{F}\left(\right.$ element of $\left.\mathfrak{F}_{\Sigma}(X)\right)=$ height $\$_{1} .\left\{\mathcal{F}\left(\tau_{1}\right): \tau_{1} \in \operatorname{rng} p\right\}$ is finite from [44, Sch. 21].

(47) Suppose $\operatorname{Arity}(o) \neq \emptyset$ and $n=\bigcup\left\{\right.$ height $\left.\tau_{1}: \tau_{1} \in \operatorname{rng} p\right\}$.

Then height $(o$-term $p)=n+1$. Proof: Set $I=\left\{\right.$ height $\tau_{1}: \tau_{1} \in \operatorname{rng} p$ \}.

$I$ is natural-membered. Define $\mathcal{F}$ (element of $\left.\mathfrak{F}_{\Sigma}(X)\right)=$ height $\$_{1} \cdot\left\{\mathcal{F}\left(\tau_{1}\right)\right.$

$\left.: \tau_{1} \in \operatorname{rng} p\right\}$ is finite from [44, Sch. 21].

(48) If $\operatorname{Arity}(o)=\emptyset$, then height $(o$-term $p)=0$.

(49) $\operatorname{deg}(x$-term $)=0$.

(50) $\operatorname{deg} \tau \neq 0$ if and only if there exists $o$ and there exists $p$ such that $\tau=o$-term $p$. Proof: Define $\mathcal{P}$ [element of $\left.\mathfrak{F}_{\Sigma}(X)\right] \equiv \operatorname{deg} \$_{1} \neq 0$ iff there exists $o$ and there exists $p$ such that $\$_{1}=o$-term $p . \mathcal{P}[x$-term $] \mathcal{P}[\tau]$ from TermInd.

Let $\tau$ be a decorated tree. Let us consider $I$. Observe that $\tau^{-1}(I)$ is finite sequence-membered.

Let us consider $a$. Let $J, K$ be sets. Let us observe that the functor $\operatorname{IFIN}(a, I$, $J, K)$ yields a set. Now we state the propositions:

(51) Suppose $J=\langle o$, the carrier of $\Sigma\rangle$. Then $(o-\operatorname{term} p)^{-1}(I)=\operatorname{IFIN}(J, I,\{\emptyset\}$, $\emptyset) \cup \bigcup\left\{\langle i\rangle \frown p(i+1)^{-1}(I): i<\operatorname{len} p\right\}$. Proof: Set $X=\left\{\langle i\rangle \frown p(i+1)^{-1}(I)\right.$ $: i<\operatorname{len} p\}$. $(o-\operatorname{term} p)^{-1}(I) \subseteq \operatorname{IFIN}(J, I,\{\emptyset\}, \emptyset) \cup \cup X$ by [20, (10)], [13, (11), (13)], [52, (25)]. 
(52) Suppose there exists a finite sequence $f$ of elements of $\mathbb{N}$ such that $i=\sum f$ and $\operatorname{dom} f=\operatorname{dom} \operatorname{Arity}(o)$ and for every $i$ and $\tau$ such that $i \in$ $\operatorname{dom} \operatorname{Arity}(o)$ and $\tau=p(i)$ holds $f(i)=\operatorname{deg} \tau$. Then $\operatorname{deg}(o-\operatorname{term} p)=i+1$. Proof: Set $\tau=o$-term $p$. Set $I=($ the carrier' of $\Sigma) \times\{$ the carrier of $\Sigma\}$. Set $\mathfrak{A}=\left\{\langle i\rangle \frown p(i+1)^{-1}(I): i<\operatorname{len} p\right\} . \emptyset \notin \bigcup \mathfrak{A} . \tau^{-1}(I)=\{\emptyset\} \cup \bigcup \mathfrak{A}$. Define $\mathcal{J}$ (natural number) $=\left\langle \$_{1}\right\rangle \frown p\left(\$_{1}+1\right)^{-1}(I)$. For every $i$ and $j$ such that $i<\operatorname{len} f$ and $j<\operatorname{len} f$ and $i \neq j$ holds $\mathcal{J}(i)$ misses $\mathcal{J}(j)$ by [22, (40)], (11). For every $i$ such that $i<\operatorname{len} f$ holds $\overline{\overline{\mathcal{J}(i)}}=f(i+1)$ by [13, (12), (13)], [52, (25)], [12, (2)]. $\overline{\overline{U\{\mathcal{J}(i): i<\operatorname{len} f\}}}=\sum f$ from CardUnion.

Let us consider $\Sigma, X, T$, and $i$. The functor $T \operatorname{deg}_{\leqslant} i$ yielding a subset of $T$ is defined by the term

(Def. 18) $\{\rho: \operatorname{deg} \rho \leqslant i\}$.

The functor $T$ height $\leqslant i$ yielding a subset of $T$ is defined by the term

(Def. 19) $\{\tau: \tau \in T$ and height $\tau \leqslant i\}$.

Now we state the propositions:

(53) $\rho \in T \operatorname{deg}_{\leqslant} i$ if and only if $\operatorname{deg} \rho \leqslant i$.

(54) $T \operatorname{deg}_{\leqslant} 0=$ the set of all $x$-term. Proof: $T \operatorname{deg}_{\leqslant} 0 \subseteq$ the set of all $x$-term by [10, (39)], (36), (50). Consider $\sigma, x$ such that $a=x$-term.

$\operatorname{deg}(x$-term $)=0 \leqslant 0$ and $x$-term $\in T$. Reconsider $\rho=x$-term as an element of $T \cdot \operatorname{deg} \rho=\operatorname{deg}{ }^{\circledR} \rho=0$.

(55) $T$ height $_{\leqslant} 0=$ the set of all $x$-term $\cup\{o$-term $p: o$-term $p \in T$ and $\operatorname{Arity}(o)=\emptyset\}$. The theorem is a consequence of (36), (46), (47), (42), and (48).

(56) $T \operatorname{deg}_{\leqslant} 0=\bigcup$ FreeGenerator $(T)$. Proof: $T \operatorname{deg}_{\leqslant} 0=$ the set of all $x$-term. $T \operatorname{deg}_{\leqslant} 0 \subseteq \cup$ FreeGenerator $(T)$ by [5, (2)]. Consider $b$ such that $b \in$ dom FreeGenerator $(T)$ and $a \in($ FreeGenerator $(T))(b)$. Consider $y$ being a set such that $y \in X(b)$ and $a=$ the root tree of $\langle y, b\rangle$.

(57) $\rho \in T$ height $_{\leqslant} i$ if and only if height $\rho \leqslant i$.

Let us consider $\Sigma, X, T$, and $i$. One can check that $T \operatorname{deg}_{\leqslant} i$ is non empty and $T$ height $_{\leqslant} i$ is non empty.

Let us assume that $i \leqslant j$. Now we state the propositions:

(58) $T \operatorname{deg}_{\leqslant} i \subseteq T \operatorname{deg}_{\leqslant} j$.

(59) $T$ height $_{\leqslant} i \subseteq T$ height $_{\leqslant} j$.

Now we state the propositions:

(60) $T \operatorname{deg}_{\leqslant}(i+1)=\left(T \operatorname{deg}_{\leqslant} 0\right) \cup\{o$-term $p$ : there exists a finite sequence $f$ of elements of $\mathbb{N}$ such that $i \geqslant \sum f$ and $\operatorname{dom} f=\operatorname{dom} \operatorname{Arity}(o)$ and for every $i$ and $\tau$ such that $i \in \operatorname{dom} \operatorname{Arity}(o)$ and $\tau=p(i)$ holds $f(i)=$ $\operatorname{deg} \tau\} \cap \bigcup($ the sorts of $T$ ). Proof: Set $I=\{o$-term $p$ : there exists a finite sequence $f$ of elements of $\mathbb{N}$ such that $i \geqslant \sum f$ and $\operatorname{dom} f=$ 
$\operatorname{dom} \operatorname{Arity}(o)$ and for every $i$ and $\tau$ such that $i \in \operatorname{dom} \operatorname{Arity}(o)$ and $\tau=$ $p(i)$ holds $f(i)=\operatorname{deg} \tau\} . T \operatorname{deg}_{\leqslant}(i+1) \subseteq\left(T \operatorname{deg}_{\leqslant} 0\right) \cup I \cap \bigcup($ the sorts of $T)$

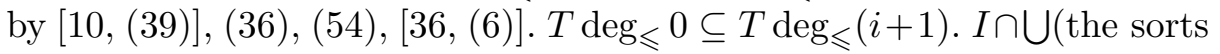
of $T) \subseteq T \operatorname{deg}_{\leqslant}(i+1)$.

(61) $T$ height $_{\leqslant}(i+1)=\left(T\right.$ height $\left._{\leqslant} 0\right) \cup\{o$-term $p: \bigcup\{$ height $\tau: \tau \in \operatorname{rng} p\} \subseteq$ $i\} \cap \bigcup($ the sorts of $T)$. Proof: Set $I=\{o$-term $p: \bigcup\{$ height $\tau: \tau \in$ $\operatorname{rng} p\} \subseteq i\}$. $T$ height $_{\leqslant}(i+1) \subseteq\left(T\right.$ height $\left._{\leqslant} 0\right) \cup I \cap \bigcup($ the sorts of $T)$ by (36), (55), (46), (47). $T$ height $_{\leqslant} 0 \subseteq T$ height $_{\leqslant}(i+1) . I \cap \bigcup$ (the sorts of $T) \subseteq T$ height $_{\leqslant}(i+1)$ by $(46),(47)$, [13, (39)], (48).

(62) $\operatorname{deg} \tau \geqslant$ height $\tau$. Proof: Define $\mathcal{P}$ [element of $\left.\mathfrak{F}_{\Sigma}(X)\right] \equiv \operatorname{deg} \$_{1} \geqslant$ height $\$_{1}$. For every operation symbol $o$ of $\Sigma$ and for every element $p$ of $\operatorname{Args}\left(o, \mathfrak{F}_{\Sigma}(X)\right)$ such that for every element $\tau$ of $\mathfrak{F}_{\Sigma}(X)$ such that $\tau \in \operatorname{rng} p$ holds $\mathcal{P}[\tau]$ holds $\mathcal{P}[o$-term $p]$ by (48), [36, (6)], (46), [42, (9)]. $\mathcal{P}[\tau]$ from TermInd.

(63) $\bigcup($ the sorts of $T)=\bigcup\left\{T \operatorname{deg}_{\leqslant} i\right.$ : not contradiction $\}$.

(64) $\bigcup($ the sorts of $T)=\bigcup\left\{T\right.$ height $_{\leqslant} i$ : not contradiction $\}$. The theorem is a consequence of (57).

(65) $T \operatorname{deg}_{\leqslant} i \subseteq \mathfrak{F}_{\Sigma}(X) \operatorname{deg}_{\leqslant} i$. Proof: Define $\mathcal{P}$ [natural number] $\equiv T \operatorname{deg}_{\leqslant} \$_{1}$ $\subseteq \mathfrak{F}_{\Sigma}(X) \operatorname{deg}_{\leqslant} \$_{1} . T \operatorname{deg}_{\leqslant} 0=\bigcup$ FreeGenerator $(T)$ and $\mathfrak{F}_{\Sigma}(X) \operatorname{deg}_{\leqslant} 0=$ $\bigcup$ FreeGenerator $\left(\mathfrak{F}_{\Sigma}(X)\right)$. For every $i, \mathcal{P}[i]$ from $[13$, Sch. 2].

\section{Context}

Let us consider $\Sigma, X, T, \sigma, x$, and $\rho$. We say that $\rho$ is $x$-context if and only if (Def. 20) $\overline{\overline{\operatorname{Coim}(\rho,\langle x, \sigma\rangle)}}=1$.

We say that $\rho$ is $x$-omitting if and only if

(Def. 21) $\operatorname{Coim}(\rho,\langle x, \sigma\rangle)=\emptyset$.

The functor vf $\rho$ yielding a set is defined by the term

(Def. 22) $\pi_{1}(\operatorname{rng} \rho \cap(\bigcup X \times($ the carrier of $\Sigma)))$.

Now we state the propositions:

(66) vf $\rho=\bigcup \operatorname{Var}_{X} \rho$. Proof: vf $\rho \subseteq \cup \operatorname{Var}_{X} \rho$ by [32, (87)], [5, (2)], [10, (44)], [23, (9)].

(67) $\operatorname{vf}(x$-term $)=\{x\}$.

(68) $\quad \operatorname{vf}(o$-term $p)=\bigcup\{\operatorname{vf} \tau: \tau \in \operatorname{rng} p\}$. Proof: $\operatorname{vf}(o$-term $p) \subseteq \bigcup\{\operatorname{vf} \tau$ $: \tau \in \operatorname{rng} p\}$ by (66), [5, (2)], [23, (13)], [55, (167)].

Let us consider $\Sigma, X, T$, and $\rho$. Note that vf $\rho$ is finite.

Now we state the proposition:

(69) If $x \notin \operatorname{vf} \rho$, then $\rho$ is $x$-omitting.

Let us consider $\Sigma, X, \sigma$, and $\tau$. We say that $\tau$ is $\sigma$-context if and only if 
(Def. 23) There exists $x$ such that $\tau$ is $x$-context.

Let us consider $x$. Let us observe that every element of $\mathfrak{F}_{\Sigma}(X)$ which is $x$-context is also $\sigma$-context.

One can verify that $x$-term is $x$-context.

One can check that there exists an element of $\mathfrak{F}_{\Sigma}(X)$ which is $x$-context and non compound and every element of $\mathfrak{F}_{\Sigma}(X)$ which is $x$-omitting is also non $x$-context.

Now we state the proposition:

(70) Let us consider sort symbols $\sigma_{1}, \sigma_{2}$ of $\Sigma$, an element $x_{1}$ of $X\left(\sigma_{1}\right)$, and an element $x_{2}$ of $X\left(\sigma_{2}\right)$. Then $\sigma_{1} \neq \sigma_{2}$ or $x_{1} \neq x_{2}$ if and only if $x_{1}$-term is $x_{2}$-omitting.

Let us consider $\Sigma, \sigma, \sigma_{1}, Z$, and $z$. Let $z^{\prime}$ be a $z$-different element of $Z\left(\sigma_{1}\right)$. One can check that $z^{\prime}$-term is $z$-omitting.

One can check that there exists an element of $\mathfrak{F}_{\Sigma}(Z)$ which is $z$-omitting.

Let us consider $\sigma_{1}$. Let $z_{1}$ be a $z$-different element of $Z\left(\sigma_{1}\right)$. Observe that there exists an element of $\mathfrak{F}_{\Sigma}(Z)$ which is $z$-omitting and $z_{1}$-context.

Let us consider $X$. Let us consider $x$.

A context of $x$ is an $x$-context element of $\mathfrak{F}_{\Sigma}(X)$. Now we state the proposition:

(71) Let us consider a sort symbol $\rho$ of $\Sigma$ and an element $y$ of $X(\rho)$. Then $x$-term is a context of $y$ if and only if $\rho=\sigma$ and $x=y$.

Let us consider $\Sigma, X$, and $\sigma$.

A context of $\sigma$ and $X$ is a $\sigma$-context element of $\mathfrak{F}_{\Sigma}(X)$. In the sequel $\mathcal{C}$ denotes a context of $x, \mathcal{C}_{1}$ denotes a context of $y, \mathcal{C}^{\prime}$ denotes a context of $z, \mathcal{C}_{11}$ denotes a context of $x_{11}, \mathcal{C}_{12}$ denotes a context of $y_{11}$, and $D$ denotes a context of $\sigma$ and $X$.

Now we state the propositions:

(72) $\mathcal{C}$ is a context of $\sigma$ and $X$.

(73) $x \in \operatorname{vf} \mathcal{C}$.

Let us consider $\Sigma, o, \sigma, X, x$, and $p$. We say that $p$ is $x$-context including once only if and only if

(Def. 24) There exists $i$ such that

(i) $i \in \operatorname{dom} p$, and

(ii) $p(i)$ is a context of $x$, and

(iii) for every $j$ and $\tau$ such that $j \in \operatorname{dom} p$ and $j \neq i$ and $\tau=p(j)$ holds $\tau$ is $x$-omitting.

Let us note that every element of $\operatorname{Args}\left(o, \mathfrak{F}_{\Sigma}(X)\right)$ which is $x$-context including once only is also non empty.

Now we state the propositions: 
(74) $p$ is $x$-context including once only if and only if $o$-term $p$ is a context of $x$. Proof: Set $I=\{\langle x, \sigma\rangle\}$. Set $k=p$. $(o \text {-term } k)^{-1}(I)=\emptyset \cup \bigcup\{\langle i\rangle \frown k(i+$ $1)^{-1}(I): i<$ len $\left.k\right\}$. If $k$ is $x$-context including once only, then $o$-term $k$ is a context of $x$ by [3, (42)], [52, (25)], [13, (10), (13), (11)].

(75) for every $i$ such that $i \in \operatorname{dom} p$ holds $p_{i}$ is $x$-omitting if and only if $o$-term $p$ is $x$-omitting. The theorem is a consequence of (51) and (13).

(76) for every $\tau$ such that $\tau \in \operatorname{rng} p$ holds $\tau$ is $x$-omitting if and only if $o$-term $p$ is $x$-omitting. The theorem is a consequence of (75).

Let us consider $\Sigma, \sigma$, and $o$. We say that $o$ is $\sigma$-dependent if and only if

(Def. 25) $\sigma \in \operatorname{rng} \operatorname{Arity}(o)$.

Let $\Sigma$ be a sufficiently rich non void non empty many sorted signature and $\sigma$ be a sort symbol of $\Sigma$. Let us note that there exists an operation symbol of $\Sigma$ which is $\sigma$-dependent.

In the sequel $\Sigma^{\prime}$ denotes a sufficiently rich non empty non void many sorted signature, $\sigma^{\prime}$ denotes a sort symbol of $\Sigma^{\prime}, o^{\prime}$ denotes a $\sigma^{\prime}$-dependent operation symbol of $\Sigma^{\prime}, X^{\prime}$ denotes a nontrivial many sorted set indexed by the carrier of $\Sigma^{\prime}$, and $x^{\prime}$ denotes an element of $X^{\prime}\left(\sigma^{\prime}\right)$.

Let us consider $\Sigma^{\prime}, \sigma^{\prime}, o^{\prime}, X^{\prime}$, and $x^{\prime}$. Let us observe that there exists an element of $\operatorname{Args}\left(o^{\prime}, \mathfrak{F}_{\Sigma^{\prime}}\left(X^{\prime}\right)\right)$ which is $x^{\prime}$-context including once only.

Let $p^{\prime}$ be an $x^{\prime}$-context including once only element of $\operatorname{Args}\left(o^{\prime}, \mathfrak{F}_{\Sigma^{\prime}}\left(X^{\prime}\right)\right)$. One can check that $o^{\prime}$-term $p^{\prime}$ is $x^{\prime}$-context.

Let us consider $\Sigma, o, \sigma, X, x$, and $p$. Assume $p$ is $x$-context including once only. The functor the $x$-context position in $p$ yielding a natural number is defined by

(Def. 26) $p(i t)$ is a context of $x$.

The functor the $x$-context in $p$ yielding a context of $x$ is defined by

(Def. 27) it $\in \operatorname{rng} p$.

Now we state the propositions:

(77) Suppose $p$ is $x$-context including once only. Then

(i) the $x$-context position in $p \in \operatorname{dom} p$, and

(ii) the $x$-context in $p=p($ the $x$-context position in $p$ ).

(78) Suppose $p$ is $x$-context including once only and the $x$-context position in $p \neq i \in \operatorname{dom} p$. Then $p_{i}$ is $x$-omitting.

Let us assume that $p$ is $x$-context including once only. Now we state the propositions:

(79) $p$ yields the $x$-context in $p$ just once. The theorem is a consequence of (77)

(80) $\quad p \leftarrow($ the $x$-context in $p)=$ the $x$-context position in $p$. The theorem is a consequence of (79). 
Now we state the proposition:

(i) $\mathcal{C}=x$-term, or

(ii) there exists $o$ and there exists $p$ such that $p$ is $x$-context including once only and $\mathcal{C}=o$-term $p$.

The theorem is a consequence of (36), (71), and (74).

Let us consider $\Sigma^{\prime}, X^{\prime}, \sigma^{\prime}$, and $x^{\prime}$. One can verify that there exists an element of $\mathfrak{F}_{\Sigma^{\prime}}\left(X^{\prime}\right)$ which is $x^{\prime}$-context and compound.

The scheme ContextInd deals with a unary predicate $\mathcal{P}$ and a non empty non void many sorted signature $\Sigma$ and a sort symbol $\sigma$ of $\Sigma$ and a non-empty many sorted set $\mathcal{X}$ indexed by the carrier of $\Sigma$ and an element $x$ of $\mathcal{X}(\sigma)$ and a context $\mathcal{C}$ of $x$ and states that

$($ Sch. 4) $\mathcal{P}[\mathcal{C}]$

provided

- $\mathcal{P}[x$-term $]$ and

- for every operation symbol $o$ of $\Sigma$ and for every element $w$ of $\operatorname{Args}\left(o, \mathfrak{F}_{\Sigma}(\mathcal{X})\right)$ such that $w$ is $x$-context including once only holds if $\mathcal{P}$ [the $x$-context in $w]$, then for every context $\mathcal{C}$ of $x$ such that $\mathcal{C}=o$-term $w$ holds $\mathcal{P}[\mathcal{C}]$.

Now we state the propositions:

(82) If $\tau$ is $x$-omitting, then $\tau_{\langle x, \sigma\rangle \leftarrow \tau_{1}}=\tau$.

(83) Suppose the sort of $\tau_{1}=\sigma$. Then $\tau_{\langle x, \sigma\rangle \leftarrow \tau_{1}} \in$ (the sorts of $\left.\mathfrak{F}_{\Sigma}(X)\right)$ (the sort of $\tau$ ). Proof: Define $\mathcal{P}$ [element of $\left.\mathfrak{F}_{\Sigma}(X)\right] \equiv \$_{1}\langle x, \sigma\rangle_{\leftarrow \tau_{1}} \in$ (the sorts of $\left.\mathfrak{F}_{\Sigma}(X)\right)$ (the sort of $\left.\$_{1}\right)$. For every $\sigma_{1}$ and for every element $y$ of $X\left(\sigma_{1}\right)$, $\mathcal{P}[y$-term $]$. For every $o$ and $p$ such that for every $\tau_{2}$ such that $\tau_{2} \in \operatorname{rng} p$ holds $\mathcal{P}\left[\tau_{2}\right]$ holds $\mathcal{P}[o$-term $p]$ by [20, (20)], (18), [52, (29)], [12, (2)]. $\mathcal{P}[\tau]$ from TermInd.

Let us consider $\Sigma, X, \sigma, x, \mathcal{C}$, and $\tau$. Assume the sort of $\tau=\sigma$. The functor $\mathcal{C}[\tau]$ yielding an element of (the sorts of $\left.\mathfrak{F}_{\Sigma}(X)\right)($ the sort of $\mathcal{C}$ ) is defined by the term

(Def. 28) $\mathcal{C}_{\langle x, \sigma\rangle \leftarrow \tau}$.

Now we state the proposition:

(84) If the sort of $\tau=\sigma$, then $x-\operatorname{term}[\tau]=\tau$.

Let us consider $\Sigma, X, \sigma, x$, and $\mathcal{C}$. Observe that $\mathcal{C}[x$-term $]$ reduces to $\mathcal{C}$.

Now we state the propositions:

(85) Let us consider an element $w$ of $\operatorname{Args}\left(o, \mathfrak{F}_{\Sigma}(Z)\right)$ and an element $\tau$ of $\mathfrak{F}_{\Sigma}(Z)$. Suppose

(i) $w$ is $z$-context including once only, and

(ii) the sort of $\tau=\operatorname{Arity}(o)$ (the $z$-context position in $w$ ). 
Then $w+\cdot($ the $z$-context position in $w, \tau) \in \operatorname{Args}\left(o, \mathfrak{F}_{\Sigma}(Z)\right)$.

(86) Suppose the sort of $\mathcal{C}^{\prime}=\sigma_{1}$. Let us consider a $z$-different element $z_{1}$ of $Z\left(\sigma_{1}\right)$ and a $z$-omitting context $\mathcal{C}_{1}$ of $z_{1}$. Then $\mathcal{C}_{1}\left[\mathcal{C}^{\prime}\right]$ is a context of $z$. PRO-

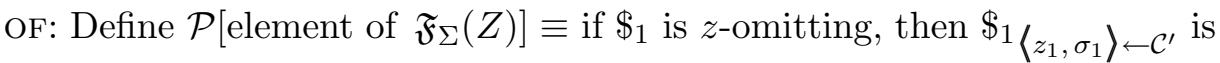
a context of $z$. For every $o$ and $k$ such that $k$ is $z_{1}$-context including once only holds if $\mathcal{P}$ [the $z_{1}$-context in $k$ ], then for every context $\mathcal{C}$ of $z_{1}$ such that $\mathcal{C}=o$-term $k$ holds $\mathcal{P}[\mathcal{C}] . \mathcal{P}\left[\mathcal{C}_{1}\right]$ from ContextInd.

(87) Let us consider elements $w, p$ of $\operatorname{Args}\left(o, \mathfrak{F}_{\Sigma}(Z)\right)$ and an element $\tau$ of $\mathfrak{F}_{\Sigma}(Z)$. Suppose

(i) $w$ is $z$-context including once only, and

(ii) $\mathcal{C}^{\prime}=o$-term $w$, and

(iii) $p=w+\cdot($ the $z$-context position in $w$, (the $z$-context in $w)[\tau])$, and

(iv) the sort of $\tau=\sigma$.

Then $\mathcal{C}^{\prime}[\tau]=o$-term $p$. The theorem is a consequence of (77), (78), (82), and (19).

(88) The sort of $\mathcal{C}[\tau]=$ the sort of $\mathcal{C}$.

(89) If $\tau(a)=\langle x, \sigma\rangle$, then $a \in \operatorname{Leaves}(\operatorname{dom} \tau)$. The theorem is a consequence of $(36)$.

(90) Let us consider a sort symbol $\sigma_{0}$ of $\Sigma$ and an element $x_{0}$ of $X\left(\sigma_{0}\right)$. Suppose

(i) the sort of $\tau=\sigma$, and

(ii) $\mathcal{C}$ is $x_{0}$-omitting, and

(iii) $\tau$ is $x_{0}$-omitting.

Then $\mathcal{C}[\tau]$ is $x_{0}$-omitting. The theorem is a consequence of (89).

(91) Suppose $p$ is $x$-context including once only. Then the sort of the $x$-context in $p=\operatorname{Arity}(o)$ (the $x$-context position in $p$ ). The theorem is a consequence of (77).

(92) Let us consider a disjoint valued non-empty algebra $\mathfrak{A}$ over $\Sigma$, a nonempty algebra $\mathcal{B}$ over $\Sigma$, an operation symbol $o$ of $\Sigma$, elements $p, q$ of $\operatorname{Args}(o, \mathfrak{A})$, a many sorted function $h$ from $\mathfrak{A}$ into $\mathcal{B}$, an element $a$ of $\mathfrak{A}$, and $i$. Suppose

(i) $i \in \operatorname{dom} p$, and

(ii) $q=p+\cdot(i, a)$.

Then $h \# q=h \# p+\cdot(i, h(a))$.

(93) Let us consider an element $\tau$ of $\mathfrak{F}_{\Sigma}(Z)$. Suppose the sort of $\tau=\sigma$. Then (the canonical homomorphism of $R)\left(\mathcal{C}^{\prime}[\tau]\right)=($ the canonical homomorphism of $R)\left(\mathcal{C}^{\prime}\left[{ }^{@}((\right.\right.$ the canonical homomorphism of $\left.\left.R)(\tau))\right]\right)$. Proof: Set $H=$ 
the canonical homomorphism of $R$. Define $\mathcal{P}$ [context of $z] \equiv H\left(\$_{1}[\tau]\right)=$ $H\left(\$_{1}\left[{ }^{\circledR}(H(\tau))\right]\right)$. The sort of ${ }^{\circledR}(H(\tau))=$ the sort of $H(\tau)$. $\mathcal{P}[z$-term $]$ by (84), [10, (48)], [28, (15)]. $\mathcal{P}\left[\mathcal{C}^{\prime}\right]$ from ContextInd.

Let us consider $\Sigma, X, T, \sigma$, and $x$. Let $h$ be a many sorted function from $\mathfrak{F}_{\Sigma}(X)$ into $T$. We say that $h$ is $x$-constant if and only if

(Def. 29) (i) $h(x$-term) $=x$-term, and

(ii) for every $\sigma_{1}$ and for every element $x_{1}$ of $X\left(\sigma_{1}\right)$ such that $x_{1} \neq x$ or $\sigma \neq \sigma_{1}$ holds $h\left(x_{1}\right.$-term $)$ is $x$-omitting.

Now we state the proposition:

(94) The canonical homomorphism of $T$ is $x$-constant. The theorem is a consequence of $(70)$.

Let us consider $\Sigma, X, T, \sigma$, and $x$. Note that there exists a homomorphism from $\mathfrak{F}_{\Sigma}(X)$ to $T$ which is $x$-constant.

From now on $h_{1}$ denotes an $x$-constant homomorphism from $\mathfrak{F}_{\Sigma}(X)$ to $T$ and $h_{2}$ denotes a $y$-constant homomorphism from $\mathfrak{F}_{\Sigma}(Y)$ to $Q$.

Let $x, y$ be objects. The functor $x \leftrightarrow y$ yielding a function is defined by the term

(Def. 30) $\{\langle x, y\rangle,\langle y, x\rangle\}$.

Let us observe that the functor is commutative.

Now we state the proposition:

(i) $\operatorname{dom}(a \leftrightarrow b)=\{a, b\}$, and

(ii) $(a \leftrightarrow b)(a)=b$, and

(iii) $(a \leftrightarrow b)(b)=a$, and

(iv) $\operatorname{rng}(a \leftrightarrow b)=\{a, b\}$.

Let $\mathfrak{A}$ be a non empty set and $a, b$ be elements of $\mathfrak{A}$. One can verify that $a \leftrightarrow b$ is $\mathfrak{A}$-valued and $\mathfrak{A}$-defined.

Let $\mathfrak{A}$ be a set, $\mathcal{B}$ be a non empty set, $f$ be a function from $\mathfrak{A}$ into $\mathcal{B}$, and $g$ be a $\mathfrak{A}$-defined $\mathcal{B}$-valued function. Let us note that the functor $f+\cdot g$ yields a function from $\mathfrak{A}$ into $\mathcal{B}$. Let $I$ be a non empty set, $\mathfrak{A}, \mathcal{B}$ be many sorted sets indexed by $I, f$ be a many sorted function from $\mathfrak{A}$ into $\mathcal{B}, x$ be an element of $I$, and $g$ be a function from $\mathfrak{A}(x)$ into $\mathcal{B}(x)$. One can verify that the functor $f+\cdot(x, g)$ yields a many sorted function from $\mathfrak{A}$ into $\mathcal{B}$. Let us consider $\Sigma, X$, $T, \sigma, x_{1}$, and $x_{2}$. The functor $\operatorname{Hom}\left(T, x_{1}, x_{2}\right)$ yielding an endomorphism of $T$ is defined by

(Def. 31) (i) it $(\sigma)\left(x_{1}\right.$-term) $=x_{2}$-term, and

(ii) it $(\sigma)\left(x_{2}\right.$-term $)=x_{1}$-term, and

(iii) for every $\sigma_{1}$ and for every element $y$ of $X\left(\sigma_{1}\right)$ such that $\sigma_{1} \neq \sigma$ or $y \neq x_{1}$ and $y \neq x_{2}$ holds $i t\left(\sigma_{1}\right)(y$-term $)=y$-term.

Now we state the propositions: 
(96) Let us consider an endomorphism $h$ of $T$. Suppose $h(\sigma)(x$-term $)=$ $x$-term. Then $h=\mathrm{id}_{\alpha}$, where $\alpha$ is the sorts of $T$. Proof: $h \uparrow$ FreeGenerator $(T)=\mathrm{id}_{\alpha} \uparrow$ FreeGenerator $(T)$, where $\alpha$ is the sorts of $T$ by [27, (49), (18)].

(97) $\operatorname{Hom}(T, x, x)=\mathrm{id}_{\alpha}$, where $\alpha$ is the sorts of $T$. The theorem is a consequence of (96).

(98) $\operatorname{Hom}\left(T, x_{1}, x_{2}\right)=\operatorname{Hom}\left(T, x_{2}, x_{1}\right)$.

(99) $\operatorname{Hom}\left(T, x_{1}, x_{2}\right) \circ \operatorname{Hom}\left(T, x_{1}, x_{2}\right)=\mathrm{id}_{\alpha}$, where $\alpha$ is the sorts of $T$. Proof: Set $h=\operatorname{Hom}\left(T, x_{1}, x_{2}\right)$. For every $\sigma$ and $x,(h \circ h)(\sigma)(x$-term $)=x$-term by [28, (15)], [36, (2)].

(100) If $\rho$ is $x_{1}$-omitting and $x_{2}$-omitting, then $\left(\operatorname{Hom}\left(T, x_{1}, x_{2}\right)\right)(\rho)=\rho$. PROOF: Define $\mathcal{P}$ [element of $T] \equiv$ if $\$_{1}$ is $x_{1}$-omitting and $x_{2}$-omitting, then $\left(\operatorname{Hom}\left(T, x_{1}, x_{2}\right)\right)\left(\right.$ the sort of $\left.\$_{1}\right)\left(\$_{1}\right)=\$_{1}$. For every $\sigma, x$, and $\rho$ such that $\rho=x$-term holds $\mathcal{P}[\rho]$. For every $o, p$, and $\rho$ such that $\rho=o$-term $p$ and for every element $\tau$ of $T$ such that $\tau \in \operatorname{rng} p$ holds $\mathcal{P}[\tau]$ holds $\mathcal{P}[\rho]$ by (22), (34), [10, (13)], [36, (6)]. $\mathcal{P}[\rho]$ from TermAlgebraInd.

Let us consider $\Sigma, X, T, \sigma$, and $x$. Let us observe that (the canonical homomorphism of $T)(\sigma)(x$-term) reduces to $x$-term.

Now we state the propositions:

(101) (The canonical homomorphism of $T$ ) $\circ \operatorname{Hom}\left(\mathfrak{F}_{\Sigma}(X), x, x_{1}\right)=\operatorname{Hom}(T, x$, $\left.x_{1}\right) \circ$ (the canonical homomorphism of $T$ ). Proof: Set $H=$ the canonical homomorphism of $T$. Set $h=\operatorname{Hom}\left(T, x, x_{1}\right)$. Set $g=\operatorname{Hom}\left(\mathfrak{F}_{\Sigma}(X), x, x_{1}\right)$. Define $\mathcal{P}$ [element of $\left.\mathfrak{F}_{\Sigma}(X)\right] \equiv(H \circ g)\left(\$_{1}\right)=(h \circ H)\left(\$_{1}\right)$. For every $\sigma$ and $x, \mathcal{P}[x$-term] by [36, (2)], [28, (15)]. For every operation symbol $o$ of $\Sigma$ and for every element $p$ of $\operatorname{Args}\left(o, \mathfrak{F}_{\Sigma}(X)\right)$ such that for every element $\tau$ of $\mathfrak{F}_{\Sigma}(X)$ such that $\tau \in \operatorname{rng} p$ holds $\mathcal{P}[\tau]$ holds $\mathcal{P}[o$-term $p]$ by [10, (13)], (34), [36, (6)], [52, (29), (25)]. $(H \circ g)(\sigma)=(h \circ H)(\sigma)$.

(102) Let us consider an element $\rho$ of $T$ from $\sigma$. Then $\left(\operatorname{Hom}\left(T, x_{1}, x_{2}\right)\right)(\sigma)(\rho)=$ ((the canonical homomorphism of $\left.T) \circ \operatorname{Hom}\left(\mathfrak{F}_{\Sigma}(X), x_{1}, x_{2}\right)\right)(\sigma)(\rho)$. The theorem is a consequence of (101).

(103) If $x_{1} \neq x_{2}$ and $\tau$ is $x_{2}$-omitting, then $\left(\operatorname{Hom}\left(\mathfrak{F}_{\Sigma}(X), x_{1}, x_{2}\right)\right)(\tau)$ is $x_{1}$ omitting. Proof: Set $T=\mathfrak{F}_{\Sigma}(X)$. Set $h=\operatorname{Hom}\left(T, x_{1}, x_{2}\right)$. Define $\mathcal{P}$ [element of $T] \equiv$ if $\$_{1}$ is $x_{2}$-omitting, then $h\left(\$_{1}\right)$ is $x_{1}$-omitting. For every $\sigma$ and $x$, $\mathcal{P}[x$-term $]$. For every $o$ and $p$ such that for every element $\tau$ of $T$ such that $\tau \in \operatorname{rng} p$ holds $\mathcal{P}[\tau]$ holds $\mathcal{P}[o-\operatorname{term} p]$ by (34), [10, (13)], [36, (6)], [12, (2)]. $\mathcal{P}[\tau]$ from TermInd.

(104) Let us consider a finite subset $\mathfrak{A}$ of $\bigcup$ (the sorts of $\left.\mathfrak{F}_{\Sigma}(Y)\right)$. Then there exists $y$ such that for every $v$ such that $v \in \mathfrak{A}$ holds $v$ is $y$-omitting. Proof: Define $\mathcal{F}$ (element of $\left.\mathfrak{F}_{\Sigma}(Y)\right)=$ vf $\$_{1} .\{\mathcal{F}(v): v \in \mathfrak{A}\}$ is finite from [44, Sch. 21]. 
Let us consider $\Sigma, X$, and $T$. We say that $T$ is structure-invariant if and only if

(Def. 32) Let us consider an element $p$ of $\operatorname{Args}(o, T)$. Suppose $(\operatorname{Den}(o, T))(p)=$ $\left(\operatorname{Den}\left(o, \mathfrak{F}_{\Sigma}(X)\right)\right)(p) \cdot(\operatorname{Den}(o, T))\left(\operatorname{Hom}\left(T, x_{1}, x_{2}\right) \# p\right)=$ $\left(\operatorname{Den}\left(o, \mathfrak{F}_{\Sigma}(X)\right)\right)\left(\operatorname{Hom}\left(T, x_{1}, x_{2}\right) \# p\right)$.

Now we state the propositions:

(105) Suppose $T$ is structure-invariant. Let us consider an element $\rho$ of $T$ from $\sigma$. Then $\left(\operatorname{Hom}\left(T, x_{1}, x_{2}\right)\right)(\sigma)(\rho)=\left(\operatorname{Hom}\left(\mathfrak{F}_{\Sigma}(X), x_{1}, x_{2}\right)\right)(\sigma)(\rho)$. Proof: Set $h=\operatorname{Hom}\left(T, x_{1}, x_{2}\right)$. Set $g=\operatorname{Hom}\left(\mathfrak{F}_{\Sigma}(X), x_{1}, x_{2}\right)$. Define $\mathcal{P}$ [element of $T] \equiv h\left(\right.$ the sort of $\left.\$_{1}\right)\left(\$_{1}\right)=g\left(\right.$ the sort of $\left.\$_{1}\right)\left(\$_{1}\right)$. For every $\sigma, x$, and $\rho$ such that $\rho=x$-term holds $\mathcal{P}[\rho]$. For every $o, p$, and $\rho$ such that $\rho=o$-term $p$ and for every element $\tau$ of $T$ such that $\tau \in \operatorname{rng} p$ holds $\mathcal{P}[\tau]$ holds $\mathcal{P}[\rho]$ by [10, (13)], (22), [36, (6)], [52, (29), (25)]. $\mathcal{P}[\rho]$ from TermAlgebraInd.

(106) If $T$ is structure-invariant and $x_{1} \neq x_{2}$ and $\rho$ is $x_{2}$-omitting, then $\left(\operatorname{Hom}\left(T, x_{1}, x_{2}\right)\right)(\rho)$ is $x_{1}$-omitting. Proof: Set $h=\operatorname{Hom}\left(T, x_{1}, x_{2}\right)$. Define $\mathcal{P}$ [element of $T] \equiv$ if $\$_{1}$ is $x_{2}$-omitting, then $h\left(\$_{1}\right)$ is $x_{1}$-omitting. For every $\sigma, x$, and $\rho$ such that $\rho=x$-term holds $\mathcal{P}[\rho]$. For every $o, p$, and $\rho$ such that $\rho=o$-term $p$ and for every element $\tau$ of $T$ such that $\tau \in \operatorname{rng} p$ holds $\mathcal{P}[\tau]$ holds $\mathcal{P}[\rho]$ by (22), (34), [10, (13), (41)]. $\mathcal{P}[\rho]$ from TermAlgebraInd.

(107) Suppose $Q$ is structure-invariant and $v$ is $y$-omitting. Then (the canonical homomorphism of $Q)(v)$ is $y$-omitting. The theorem is a consequence of (104), (29), (101), (100), (98), and (106).

(108) Suppose $Q$ is structure-invariant. Let us consider an element $p$ of $\operatorname{Args}(o, Q)$. Suppose an element $\tau$ of $Q$. If $\tau \in \operatorname{rng} p$, then $\tau$ is $y$-omitting. Let us consider an element $\tau$ of $Q$. If $\tau=(\operatorname{Den}(o, Q))(p)$, then $\tau$ is $y$-omitting. The theorem is a consequence of (76), (34), and (107).

(109) If $Q$ is structure-invariant and $v$ is $y$-omitting, then $h_{2}(v)$ is $y$-omitting. Proof: Define $\mathcal{P}$ [element of $\left.\mathfrak{F}_{\Sigma}(Y)\right] \equiv$ if $\$_{1}$ is $y$-omitting, then $h_{2}\left(\$_{1}\right)$ is $y$-omitting. For every $\sigma$ and $y, \mathcal{P}[y$-term $]$. For every $o$ and $q$ such that for every $v$ such that $v \in \operatorname{rng} q$ holds $\mathcal{P}[v]$ holds $\mathcal{P}[o$-term $q]$ by (34), [10, (13)], [36, (6)], [12, (2)]. $\mathcal{P}[v]$ from TermInd.

Let us consider a terminating invariant stable many sorted relation $R$ indexed by $\mathfrak{F}_{\Sigma}(X)$ with NF-variables and unique normal form property. Now we state the propositions:

(110) (i) for every element $\tau$ of the algebra of normal forms of $R,\left(\operatorname{Hom}\left(\mathfrak{F}_{\Sigma}(X)\right.\right.$, $\left.x_{1}, x_{2}\right)$ )(the sort of $\left.\tau\right)(\tau)=($ Hom(the algebra of normal forms of $\left.\left.R, x_{1}, x_{2}\right)\right)(\tau)$, and

(ii) $\operatorname{Hom}\left(\mathfrak{F}_{\Sigma}(X), x_{1}, x_{2}\right) \uparrow \operatorname{NForms}(R)=\operatorname{Hom}$ (the algebra of normal 
forms of $\left.R, x_{1}, x_{2}\right)$.

Proof: Set $F=\mathfrak{F}_{\Sigma}(X)$. Set $T=$ the algebra of normal forms of $R$. Set $H_{3}=\operatorname{Hom}\left(F, x_{1}, x_{2}\right)$. Set $H_{2}=\operatorname{Hom}\left(T, x_{1}, x_{2}\right)$. Define $\mathcal{P}$ [element of $\left.T\right] \equiv$ $H_{3}$ (the sort of $\left.\$_{1}\right)\left(\$_{1}\right)=H_{2}\left(\$_{1}\right)$. For every sort symbol $\sigma$ of $\Sigma$ and for every element $x$ of $X(\sigma)$ and for every element $\rho$ of $T$ such that $\rho=x$-term holds $\mathcal{P}[\rho]$. For every operation symbol $o$ of $\Sigma$ and for every element $p$ of $\operatorname{Args}\left(o, \mathfrak{F}_{\Sigma}(X)\right)$ and for every element $\rho$ of $T$ such that $\rho=o$-term $p$ and for every element $\tau$ of $T$ such that $\tau \in \operatorname{rng} p$ holds $\mathcal{P}[\tau]$ holds $\mathcal{P}[\rho]$ by (22), (34), [10, (13)], [16, (54)]. $\left(\operatorname{Hom}\left(\mathfrak{F}_{\Sigma}(X), x_{1}, x_{2}\right) \uparrow \operatorname{NForms}(R)\right)(\sigma)=$ (Hom(the algebra of normal forms of $\left.\left.R, x_{1}, x_{2}\right)\right)(\sigma)$ by [27, (49)].

(111) Suppose $i \in \operatorname{dom} p$ and $R\left(\operatorname{Arity}(o)_{i}\right)$ reduces $\tau_{1}$ to $\tau_{2}$. Then $R$ (the result sort of $o$ ) reduces $\left(\operatorname{Den}\left(o, \mathfrak{F}_{\Sigma}(X)\right)\right)\left(p+\cdot\left(i, \tau_{1}\right)\right)$ to $\left(\operatorname{Den}\left(o, \mathfrak{F}_{\Sigma}(X)\right)\right)(p+\cdot$ $\left.\left(i, \tau_{2}\right)\right)$. Proof: Consider $\rho$ being a reduction sequence w.r.t. $R\left(\operatorname{Arity}(o)_{i}\right)$ such that $\rho(1)=\tau_{1}$ and $\rho(\operatorname{len} \rho)=\tau_{2}$. Define $\mathcal{P}$ [natural number] $\equiv$ if $\$_{1} \leqslant$ len $\rho$, then $R$ (the result sort of $o$ ) reduces $\left(\operatorname{Den}\left(o, \mathfrak{F}_{\Sigma}(X)\right)\right)\left(p+\cdot\left(i, \tau_{1}\right)\right)$ to $\left(\operatorname{Den}\left(o, \mathfrak{F}_{\Sigma}(X)\right)\right)\left(p+\cdot\left(i, \rho\left(\$_{1}\right)\right)\right)$. For every $i$ such that $1 \leqslant i$ and $\mathcal{P}[i]$ holds $\mathcal{P}[i+1]$ by [13, (13)], [52, (25)], [32, (87)], [12, (7), (2)]. For every $i$ such that $i \geqslant 1$ holds $\mathcal{P}[i]$ from [13, Sch. 8].

Now we state the propositions:

(112) Let us consider a terminating invariant stable many sorted relation $R$ indexed by $\mathfrak{F}_{\Sigma}(X)$ with $\mathrm{NF}$-variables and unique normal form property and $\tau$. Then $R$ (the sort of $\tau$ ) reduces $\tau$ to (the canonical homomorphism of the algebra of normal forms of $R)(\tau)$. Proof: Set $T=$ the algebra of normal forms of $R$. Set $H=$ the canonical homomorphism of $T$. Define $\mathcal{P}$ [element of $\left.\mathfrak{F}_{\Sigma}(X)\right] \equiv R$ (the sort of $\left.\$_{1}\right)$ reduces $\$_{1}$ to $H\left(\$_{1}\right)$. For every $o$ and $p$ such that for every $\tau$ such that $\tau \in \operatorname{rng} p$ holds $\mathcal{P}[\tau]$ holds $\mathcal{P}[o$-term $p]$ by [10, (13)], (34), [16, (54)], [12, (2)]. $\mathcal{P}[\tau]$ from TermInd.

(113) Let us consider a terminating invariant stable many sorted relation $R$ indexed by $\mathfrak{F}_{\Sigma}(X)$ with $\mathrm{NF}$-variables and unique normal form property, $o$, and $p$. Then $R$ (the result sort of $o$ ) reduces $o$-term $p$ to (Den $(o$, the algebra of normal forms of $R)$ )((the canonical homomorphism of the algebra of normal forms of $R$ )\#p). The theorem is a consequence of (34) and (112).

(114) Let us consider a terminating invariant stable many sorted relation $R$ indexed by $\mathfrak{F}_{\Sigma}(X)$ with NF-variables and unique normal form property, $o$, $p$, and an element $q$ of $\operatorname{Args}(o$, the algebra of normal forms of $R$ ). Suppose $p=q$. Then $R$ (the result sort of $o$ ) reduces $o$-term $p$ to (Den $(o$, the algebra of normal forms of $R))(q)$. The theorem is a consequence of (113).

Let us consider $\Sigma$ and $X$. Let $R$ be a terminating invariant stable many sorted relation indexed by $\mathfrak{F}_{\Sigma}(X)$ with $\mathrm{NF}$-variables and unique normal form property. Observe that the algebra of normal forms of $R$ is structure-invariant.

Let us note that there exists a free in itself including $\Sigma$-terms over $X$ algebra 
over $\Sigma$ with all variables and inheriting operations which is structure-invariant.

\section{Context vs. Translations}

Let us consider $\Sigma, \sigma_{1}$, and $\sigma_{2}$. We say that $\sigma_{2}$ is $\sigma_{1}$-reachable if and only if (Def. 33) TranslRel $(\Sigma)$ reduces $\sigma_{1}$ to $\sigma_{2}$.

One can verify that there exists a sort symbol of $\Sigma$ which is $\sigma_{1}$-reachable.

From now on $\sigma_{2}$ denotes a $\sigma_{1}$-reachable sort symbol of $\Sigma$ and $g_{1}$ denotes a translation in $\mathfrak{F}_{\Sigma}(Y)$ from $\sigma_{1}$ into $\sigma_{2}$.

Now we state the proposition:

(115) TranslRel $(\Sigma)$ reduces $\sigma$ to the sort of $\mathcal{C}^{\prime}$. Proof: Define $\mathcal{P}$ [element of $\left.\mathfrak{F}_{\Sigma}(Z)\right] \equiv \operatorname{TranslRel}(\Sigma)$ reduces $\sigma$ to the sort of $\$_{1} \cdot \mathcal{P}\left[\mathcal{C}^{\prime}\right]$ from ContextInd.

Let us consider $\Sigma, X, \sigma, x$, and $\mathcal{C}$. Observe that the sort of $\mathcal{C}$ is $\sigma$-reachable.

Let us consider $\sigma_{1}, \sigma_{2}$, and $g$. Let $\tau$ be an element of (the sorts of $\left.\mathfrak{F}_{\Sigma}(X)\right)\left(\sigma_{1}\right)$. One can check that the functor $g(\tau)$ yields an element of (the sorts of $\left.\mathfrak{F}_{\Sigma}(X)\right)\left(\sigma_{2}\right)$. Let us consider $\sigma, x$, and $\mathcal{C}$. We say that $\mathcal{C}$ is basic if and only if

(Def. 34) There exists $o$ and there exists $p$ such that $\mathcal{C}=o$-term $p$ and the $x$-context in $p=x$-term.

The functor transl $\mathcal{C}$ yielding a function from (the sorts of $\left.\mathfrak{F}_{\Sigma}(X)\right)(\sigma)$ into (the sorts of $\mathfrak{F}_{\Sigma}(X)$ )(the sort of $\mathcal{C}$ ) is defined by

(Def. 35) If the sort of $\tau=\sigma$, then it $(\tau)=\mathcal{C}[\tau]$.

Now we state the propositions:

(116) If $\mathcal{C}=x$-term, then transl $\mathcal{C}=\mathrm{id}_{\alpha(\sigma)}$, where $\alpha$ is the sorts of $\mathfrak{F}_{\Sigma}(X)$. The theorem is a consequence of $(84)$.

(117) Suppose $\mathcal{C}^{\prime}=o$-term $k$ and the $z$-context in $k=z$-term and $k 1=$ $k+\cdot($ the $z$-context position in $k, l)$. Then $\mathcal{C}^{\prime}[l]=o$-term $k 1$. The theorem is a consequence of $(74),(77),(84)$, and (87).

(118) If $\mathcal{C}^{\prime}$ is basic, then transl $\mathcal{C}^{\prime}$ is an elementary translation in $\mathfrak{F}_{\Sigma}(Z)$ from $\sigma$ into the sort of $\mathcal{C}^{\prime}$. The theorem is a consequence of $(34),(74),(77)$, and (117).

(119) Let us consider a finite set $V$. Suppose

(i) $m \in \operatorname{dom} q$, and

(ii) $\operatorname{Arity}(o)_{m}=\sigma$.

Then there exists $y$ and there exists $\mathcal{C}_{1}$ and there exists $q_{1}$ such that $y \notin V$ and $\mathcal{C}_{1}=o$-term $q_{1}$ and $q_{1}=q+\cdot(m, y$-term $)$ and $q_{1}$ is $y$-context including once only and $m=$ the $y$-context position in $q_{1}$ and the $y$-context in $q_{1}=$ $y$-term. Proof: Set $y=$ the element of $Y(\sigma) \backslash\left(V \cup \pi_{1}(\operatorname{rng}(o\right.$-term $\left.q))\right)$. 
Reconsider $q_{1}=q+\cdot(m, y$-term $)$ as an element of $\operatorname{Args}\left(o, \mathfrak{F}_{\Sigma}(Y)\right) \cdot q_{1}$ is $y$-context including once only by [25, (30), (31), (32)], [52, (25)].

(120) Let us consider sort symbols $\sigma_{1}, \sigma_{2}$ of $\Sigma$ and a finite set $V$. Suppose

(i) $m \in \operatorname{dom} q$, and

(ii) $\sigma_{1}=\operatorname{Arity}(o)_{m}$.

Then there exists an element $y$ of $Y\left(\sigma_{1}\right)$ and there exists a context $\mathcal{C}$ of $y$ and there exists $q_{1}$ such that $y \notin V$ and $q_{1}=q+\cdot(m, y$-term $)$ and $q_{1}$ is $y$-context including once only and the $y$-context in $q_{1}=y$-term and $\mathcal{C}=o$-term $q_{1}$ and $m=$ the $y$-context position in $q_{1}$ and transl $\mathcal{C}=$ $o_{m}^{\mathfrak{F}_{\Sigma}(Y)}(q,-)$. The theorem is a consequence of (119) and (117).

Let us consider $\Sigma, X, \tau$, and $a$. One can verify that $\operatorname{Coim}(\tau, a)$ is finite sequence-membered.

Now we state the propositions:

(121) Suppose $X$ is nontrivial and the sort of $\tau=\sigma$. Then $\overline{\overline{\operatorname{Coim}(\tau, a)}} \subseteq$ $\overline{\overline{\operatorname{Coim}(\mathcal{C}[\tau], a)}}$. Proof: Define $\mathcal{P}[$ context of $x] \equiv$ for every $\mathcal{C}$ such that

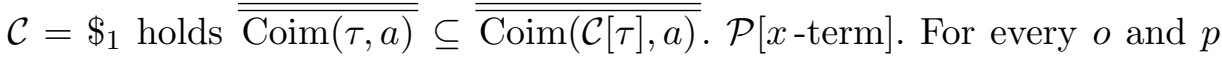
such that $p$ is $x$-context including once only holds if $\mathcal{P}$ [the $x$-context in $p$ ], then for every context $\mathcal{C}$ of $x$ such that $\mathcal{C}=o$-term $p$ holds $\mathcal{P}[\mathcal{C}]$ by $(77)$, [36, (6)], [13, (10)], [52, (25)]. $\mathcal{P}[\mathcal{C}]$ from ContextInd.

(122) If $p$ is $x$-context including once only and $i \in \operatorname{dom} p$, then $p_{i}$ is not $x$ omitting iff $p_{i}$ is $x$-context.

Let us assume that $X$ is nontrivial and the sort of $\mathcal{C}=\sigma_{1}$. Now we state the propositions:

(123) Let us consider an element $x_{1}$ of $X\left(\sigma_{1}\right)$, a context $\mathcal{C}_{1}$ of $x_{1}$, and a context $\mathcal{C}_{2}$ of $x$. Suppose $\mathcal{C}_{2}=\mathcal{C}_{1}[\mathcal{C}]$. If the sort of $\tau=\sigma$, then $\mathcal{C}_{2}[\tau]=\mathcal{C}_{1}[\mathcal{C}[\tau]]$. PRoOF: Define $\mathcal{P}$ [context of $\left.x_{1}\right] \equiv$ for every context $\mathcal{C}_{1}$ of $x_{1}$ for every context $\mathcal{C}_{2}$ of $x$ such that $\mathcal{C}_{1}=\$_{1}$ and $\mathcal{C}_{2}=\mathcal{C}_{1}[\mathcal{C}]$ holds $\mathcal{C}_{2}[\tau]=\mathcal{C}_{1}[\mathcal{C}[\tau]]$. $\mathcal{P}\left[x_{1}\right.$-term]. For every $o$ and for every element $w$ of $\operatorname{Args}\left(o, \mathfrak{F}_{\Sigma}(X)\right)$ such that $w$ is $x_{1}$-context including once only holds if $\mathcal{P}$ [the $x_{1}$-context in $w$ ], then for every context $\mathcal{C}$ of $x_{1}$ such that $\mathcal{C}=o$-term $w$ holds $\mathcal{P}[\mathcal{C}]$ by $(77)$, [36. (6)], [12, (2), (7)]. $\mathcal{P}\left[\mathcal{C}_{1}\right]$ from ContextInd.

(124) Let us consider an element $x_{1}$ of $X\left(\sigma_{1}\right)$, a context $\mathcal{C}_{1}$ of $x_{1}$, and a context $\mathcal{C}_{2}$ of $x$. Suppose $\mathcal{C}_{2}=\mathcal{C}_{1}[\mathcal{C}]$. Then transl $\mathcal{C}_{2}=\operatorname{transl} \mathcal{C}_{1} \cdot \operatorname{transl} \mathcal{C}$. Proof: Reconsider $f=\operatorname{transl} \mathcal{C}$ as a function from (the sorts of $\left.\mathfrak{F}_{\Sigma}(X)\right)(\sigma)$ into (the sorts of $\left.\mathfrak{F}_{\Sigma}(X)\right)\left(\sigma_{1}\right) \cdot \operatorname{transl} \mathcal{C}_{2}=\operatorname{transl} \mathcal{C}_{1} \cdot f$ by [28, (15)], (123).

Now we state the proposition:

(125) There exists $y_{11}$ and there exists $\mathcal{C}_{12}$ such that the sort of $\mathcal{C}_{12}=\sigma_{2}$ and $g_{1}=\operatorname{transl} \mathcal{C}_{12}$. Proof: Define $\mathcal{P}$ [function, sort symbol of $\Sigma$, sort symbol of $\Sigma] \equiv$ for every finite set $V$, there exists an element $x$ of $Y\left(\$_{2}\right)$ and 
there exists a context $\mathcal{C}$ of $x$ such that $x \notin V$ and the sort of $\mathcal{C}=\$_{3}$ and $\$_{1}=\operatorname{transl} \mathcal{C}$. For every $\sigma, \mathcal{P}\left[\operatorname{id}_{\alpha(\sigma)}, \sigma, \sigma\right]$, where $\alpha$ is the sorts of $\mathfrak{F}_{\Sigma}(Y)$. For every sort symbols $\sigma_{1}, \sigma_{2}, \sigma_{3}$ of $\Sigma$ such that $\operatorname{TranslRel}(\Sigma)$ reduces $\sigma_{1}$ to $\sigma_{2}$ for every translation $\tau$ in $\mathfrak{F}_{\Sigma}(Y)$ from $\sigma_{1}$ into $\sigma_{2}$ such that $\mathcal{P}\left[\tau, \sigma_{1}, \sigma_{2}\right]$ for every function $f$ such that $f$ is an elementary translation in $\mathfrak{F}_{\Sigma}(Y)$ from $\sigma_{2}$ into $\sigma_{3}$ holds $\mathcal{P}\left[f \cdot \tau, \sigma_{1}, \sigma_{3}\right]$ by [12, (2)], (120), (73), (69). For every sort symbols $\sigma_{1}, \sigma_{2}$ of $\Sigma$ such that TranslRel $(\Sigma)$ reduces $\sigma_{1}$ to $\sigma_{2}$ for every translation $\tau$ in $\mathfrak{F}_{\Sigma}(Y)$ from $\sigma_{1}$ into $\sigma_{2}, \mathcal{P}\left[\tau, \sigma_{1}, \sigma_{2}\right]$ from [12, Sch. 1].

The scheme LambdaTerm deals with a non empty non void many sorted signature $\Sigma$ and a non-empty many sorted set $\mathcal{X}$ indexed by the carrier of $\Sigma$ and including $\Sigma$-terms over $\mathcal{X}$ algebras $T_{1}, T_{2}$ over $\Sigma$ with all variables and inheriting operations and a unary functor $\mathcal{F}$ yielding an element of $T_{2}$ and states that

(Sch. 5) There exists a many sorted function $f$ from $T_{1}$ into $T_{2}$ such that for every element $\tau$ of $T_{1}, f(\tau)=\mathcal{F}(\tau)$

provided

- for every element $\tau$ of $T_{1}$, the sort of $\tau=$ the sort of $\mathcal{F}(\tau)$.

Now we state the propositions:

(126) There exists an endomorphism $g$ of $T$ such that

(i) (the canonical homomorphism of $T$ ) $\circ h=$ $g \circ$ (the canonical homomorphism of $T$ ), and

(ii) for every element $\tau$ of $T, g(\tau)=$ (the canonical homomorphism of $T)\left(h\left({ }^{\Theta} \tau\right)\right)$.

The theorem is a consequence of (29).

(127) (The canonical homomorphism of $T)(h(\tau))=$

(the canonical homomorphism of $T)\left(h\left({ }^{@}((\right.\right.$ the canonical homomorphism of $T(\tau))))$. The theorem is a consequence of (126) and (29).

\section{Context vs. Endomorphism}

Let us consider $\Sigma$. Let $\mathcal{B}$ be a non empty finite sequence of elements of the carrier of $\Sigma$ and $i$ be an element of $\operatorname{dom} \mathcal{B}$. Note that the functor $\mathcal{B}(i)$ yields a sort symbol of $\Sigma$. Let us consider $X$. Let $\mathcal{B}$ be a finite sequence of elements of the carrier of $\Sigma$ and $V$ be a finite sequence of elements of $\bigcup X$. We say that $V$ is $\mathcal{B}$-sorting if and only if

(Def. 36) (i) $\operatorname{dom} V=\operatorname{dom} \mathcal{B}$, and

(ii) for every $i$ such that $i \in \operatorname{dom} \mathcal{B}$ holds $V(i) \in X(\mathcal{B}(i))$. 
Let us observe that there exists a finite sequence of elements of $\bigcup X$ which is $\mathcal{B}$-sorting.

Let $\mathcal{B}$ be a non empty finite sequence of elements of the carrier of $\Sigma$. One can check that every finite sequence of elements of $\bigcup X$ which is $\mathcal{B}$-sorting is also non empty.

Let $V$ be a $\mathcal{B}$-sorting finite sequence of elements of $\bigcup X$ and $i$ be an element of $\operatorname{dom} \mathcal{B}$. Note that the functor $V(i)$ yields an element of $X(\mathcal{B}(i))$. Let $\mathcal{B}$ be a finite sequence of elements of the carrier of $\Sigma$ and $D$ be a finite sequence of elements of $\mathfrak{F}_{\Sigma}(X)$. We say that $D$ is $\mathcal{B}$-sorting if and only if

(Def. 37) (i) $\operatorname{dom} D=\operatorname{dom} \mathcal{B}$, and

(ii) for every $i$ such that $i \in \operatorname{dom} \mathcal{B}$ holds $D(i) \in\left(\right.$ the sorts of $\left.\mathfrak{F}_{\Sigma}(X)\right)(\mathcal{B}(i))$.

Note that there exists a finite sequence of elements of $\mathfrak{F}_{\Sigma}(X)$ which is $\mathcal{B}$ sorting.

Let $\mathcal{B}$ be a non empty finite sequence of elements of the carrier of $\Sigma$. One can verify that every finite sequence of elements of $\mathfrak{F}_{\Sigma}(X)$ which is $\mathcal{B}$-sorting is also non empty.

Let $D$ be a $\mathcal{B}$-sorting finite sequence of elements of $\mathfrak{F}_{\Sigma}(X)$ and $i$ be an element of $\operatorname{dom} \mathcal{B}$. Let us note that the functor $D(i)$ yields an element of (the sorts of $\left.\mathfrak{F}_{\Sigma}(X)\right)(\mathcal{B}(i))$. Let $V$ be a $\mathcal{B}$-sorting finite sequence of elements of $\bigcup X$ and $F$ be a finite sequence of elements of $\mathfrak{F}_{\Sigma}(X)$. We say that $F$ is $V$-context sequence if and only if

(Def. 38) (i) $\operatorname{dom} F=\operatorname{dom} \mathcal{B}$, and

(ii) for every element $i$ of $\operatorname{dom} \mathcal{B}, F(i)$ is a context of $V(i)$.

Let us observe that every finite sequence of elements of $\mathfrak{F}_{\Sigma}(X)$ which is $V$-context sequence is also non empty.

The scheme FinSeqLambda deals with a non empty finite sequence $\mathcal{B}$ and a unary functor $\mathcal{F}$ yielding an object and states that

(Sch. 6) There exists a non empty finite sequence $p$ such that $\operatorname{dom} p=\operatorname{dom} \mathcal{B}$ and for every element $i$ of $\operatorname{dom} \mathcal{B}, p(i)=\mathcal{F}(i)$.

The scheme FinSeqRecLambda deals with a non empty finite sequence $\mathcal{B}$ and an object $\mathfrak{A}$ and a binary functor $\mathcal{F}$ yielding a set and states that

(Sch. 7) There exists a non empty finite sequence $p$ such that $\operatorname{dom} p=\operatorname{dom} \mathcal{B}$ and $p(1)=\mathfrak{A}$ and for every elements $i, j$ of $\operatorname{dom} \mathcal{B}$ such that $j=i+1$ holds $p(j)=\mathcal{F}(i, p(i))$.

The scheme FinSeqRec2Lambda deals with a non empty finite sequence $\mathcal{B}$ and a decorated tree $\mathfrak{A}$ and a binary functor $\mathcal{F}$ yielding a decorated tree and states that

(Sch. 8) There exists a non empty decorated tree yielding finite sequence $p$ such that $\operatorname{dom} p=\operatorname{dom} \mathcal{B}$ and $p(1)=\mathfrak{A}$ and for every elements $i, j$ of $\operatorname{dom} \mathcal{B}$ 
such that $j=i+1$ for every decorated tree $d$ such that $d=p(i)$ holds $p(j)=\mathcal{F}(i, d)$.

Let us consider $\Sigma$ and $X$. Let $\mathcal{B}$ be a non empty finite sequence of elements of the carrier of $\Sigma$ and $V$ be a $\mathcal{B}$-sorting finite sequence of elements of $\bigcup X$. One can check that there exists a finite sequence of elements of $\mathfrak{F}_{\Sigma}(X)$ which is $V$-context sequence.

Let $F$ be a $V$-context sequence finite sequence of elements of $\mathfrak{F}_{\Sigma}(X)$ and $i$ be an element of $\operatorname{dom} \mathcal{B}$. One can verify that the functor $F(i)$ yields a context of $V(i)$. Let $V_{1}, V_{2}$ be $\mathcal{B}$-sorting finite sequences of elements of $\bigcup X$. We say that $V_{2}$ is $V_{1}$-omitting if and only if

(Def. 39) $\operatorname{rng} V_{1}$ misses $\operatorname{rng} V_{2}$.

Let $D$ be a $\mathcal{B}$-sorting finite sequence of elements of $\mathfrak{F}_{\Sigma}(X)$ and $F$ be a $V_{2^{-}}$ context sequence finite sequence of elements of $\mathfrak{F}_{\Sigma}(X)$. We say that $F$ is $\left(V_{1}\right.$, $\left.V_{2}, D\right)$-consequent context sequence if and only if

(Def. 40) Let us consider elements $i, j$ of $\operatorname{dom} \mathcal{B}$. If $i+1=j$, then $F(j)\left[V_{1}(j)\right.$-term $]=$ $F(i)[D(i)]$.

Let $V$ be a $\mathcal{B}$-sorting finite sequence of elements of $\bigcup X$. We say that $V$ is $D$-omitting if and only if

(Def. 41) If $\tau \in \operatorname{rng} D$, then vf $\tau$ misses $\operatorname{rng} V$.

Now we state the proposition:

(128) Let us consider a non empty finite sequence $\mathcal{B}$ of elements of the carrier of $\Sigma$ a $\mathcal{B}$-sorting finite sequence $D$ of elements of $\mathfrak{F}_{\Sigma}(X)$ a $\mathcal{B}$-sorting finite sequence $V$ of elements of $\bigcup X$. Suppose $V$ is $D$-omitting. Let us consider elements $b_{1}, b_{2}$ of dom $\mathcal{B}$. Then $D\left(b_{1}\right)$ is $\left(V\left(b_{2}\right)\right)$-omitting. The theorem is a consequence of (69).

Let us consider $\Sigma$ and $Y$. Let $\mathcal{B}$ be a non empty finite sequence of elements of the carrier of $\Sigma, V$ be a $\mathcal{B}$-sorting finite sequence of elements of $\bigcup Y$, and $D$ be a $\mathcal{B}$-sorting finite sequence of elements of $\mathfrak{F}_{\Sigma}(Y)$. Let us observe that there exists a $\mathcal{B}$-sorting finite sequence of elements of $\bigcup Y$ which is one-to-one, $V$-omitting, and $D$-omitting.

Let us consider $X$ and $\tau$.

A vf-sequence of $\tau$ is a finite sequence and is defined by

(Def. 42) There exists a one-to-one finite sequence $f$ such that

(i) $\operatorname{rng} f=\{\xi$, where $\xi$ is an element of $\operatorname{dom} \tau$ : there exists $\sigma$ and there exists $x$ such that $\tau(\xi)=\langle x, \sigma\rangle\}$, and

(ii) $\operatorname{dom} i t=\operatorname{dom} f$, and

(iii) for every $i$ such that $i \in \operatorname{dom} i t$ holds $i t(i)=\tau(f(i))$.

Let $f$ be a finite sequence. Let us observe that $\operatorname{pr} 1(f)$ is finite sequence-like and $\operatorname{pr} 2(f)$ is finite sequence-like.

Now we state the propositions: 
(129) Let us consider a vf-sequence $f$ of $\tau$. Then $\operatorname{pr} 2(f)$ is a finite sequence of elements of the carrier of $\Sigma$.

(130) Let us consider a vf-sequence $f$ of $\tau$ and a finite sequence $\mathcal{B}$ of elements of the carrier of $\Sigma$. Suppose $\mathcal{B}=\operatorname{pr} 2(f)$. Then $\operatorname{pr} 1(f)$ is a $\mathcal{B}$-sorting finite sequence of elements of $\bigcup X$.

Let $f$ be a non empty finite sequence. One can verify that $1(\in \operatorname{dom} f)$ reduces to 1 and $(\operatorname{len} f)(\in \operatorname{dom} f)$ reduces to $\operatorname{len} f$.

Now we state the propositions:

(131) Let us consider an element $\xi$ of $\operatorname{dom} \tau$. Suppose $\tau(\xi)=\langle x, \sigma\rangle$. Suppose the sort of $\tau_{1}=\sigma$. Then $\tau$ with-replacement $\left(\xi, \tau_{1}\right)$ is an element of $\mathfrak{F}_{\Sigma}(X)$ from the sort of $\tau$. Proof: Define $\mathcal{P}$ [element of $\left.\mathfrak{F}_{\Sigma}(X)\right] \equiv$ for every element $\xi$ of $\operatorname{dom} \$_{1}$ for every $x_{1}$ and $\tau$ such that $\$_{1}(\xi)=\left\langle x_{1}, \sigma\right\rangle$ and $\tau=\$_{1}$ holds $\$_{1}$ with-replacement $\left(\xi, \tau_{1}\right)$ is an element of $\mathfrak{F}_{\Sigma}(X)$ from the sort of $\tau$. $\mathcal{P}\left[x_{11}\right.$-term] by [20, (3)], [17, (29)]. For every $o$ and $p$ such that for every $\tau$ such that $\tau \in \operatorname{rng} p$ holds $\mathcal{P}[\tau]$ holds $\mathcal{P}[o$-term $p]$ by [20, (10)], [13, (12), (13)], [52, (25)]. $\mathcal{P}[\tau]$ from TermInd.

(132) Suppose $X$ is nontrivial. Let us consider an element $\xi$ of dom $\mathcal{C}$. Suppose $\mathcal{C}(\xi)=\langle x, \sigma\rangle$. If the sort of $\tau=\sigma$, then $\mathcal{C}[\tau]=\mathcal{C}$ with-replacement $(\xi, \tau)$. Proof: Define $\mathcal{P}$ [element of $\left.\mathfrak{F}_{\Sigma}(X)\right] \equiv$ for every context $\mathcal{C}$ of $x$ such that $\mathcal{C}=\$_{1}$ for every element $\xi$ of $\operatorname{dom} \mathcal{C}$ such that $\mathcal{C}(\xi)=\langle x, \sigma\rangle$ holds $\mathcal{C}[\tau]=\mathcal{C}$ with-replacement $(\xi, \tau) . \mathcal{P}[x$-term] by [17, (29)], [20, (3)], (84). For every operation symbol $o$ of $\Sigma$ and for every element $w$ of $\operatorname{Args}\left(o, \mathfrak{F}_{\Sigma}(X)\right)$ such that $w$ is $x$-context including once only holds if $\mathcal{P}$ [the $x$-context in $w]$, then for every context $\mathcal{C}$ of $x$ such that $\mathcal{C}=o$-term $w$ holds $\mathcal{P}[\mathcal{C}]$ by [20, (10)], [19, (38)], [13, (12), (13)]. $\mathcal{P}[\mathcal{C}]$ from ContextInd.

(133) Let us consider finite sequences $\xi_{1}, \xi_{2}$. Suppose

(i) $\xi_{1} \neq \xi_{2}$, and

(ii) $\xi_{1}, \xi_{2} \in \operatorname{dom} \tau$.

Let us consider sort symbols $\sigma_{1}, \sigma_{2}$ of $\Sigma$, an element $x_{1}$ of $X\left(\sigma_{1}\right)$, and an element $x_{2}$ of $X\left(\sigma_{2}\right)$. Suppose $\tau\left(\xi_{1}\right)=\left\langle x_{1}, \sigma_{1}\right\rangle$. Then $\xi_{1} \npreceq \xi_{2}$. The theorem is a consequence of $(36)$.

Let us consider $\tau, \tau_{1}$, and an element $\xi$ of $\operatorname{dom} \tau$. Now we state the propositions:

(134) If $\tau_{1}=\tau$ with-replacement $\left(\xi, x\right.$-term) and $\tau$ is $x$-omitting, then $\tau_{1}$ is a context of $x$. Proof: $\operatorname{Coim}\left(\tau_{1},\langle x, \sigma\rangle\right)=\{\xi\}$ by [17, (1), (29)], [20, (3)], [22, (87)].

(135) If $\tau(\xi)=\langle x, \sigma\rangle$, then $\operatorname{dom} \tau \subseteq \operatorname{dom}\left(\tau\right.$ with-replacement $\left.\left(\xi, \tau_{1}\right)\right)$. The theorem is a consequence of (89).

Now we state the propositions: 
(136) Let us consider an element $\xi$ of $\operatorname{dom} \tau$. Suppose $\tau(\xi)=\langle x, \sigma\rangle$. Then $\operatorname{dom} \tau=\operatorname{dom}\left(\tau\right.$ with-replacement $\left(\xi, x_{1}\right.$-term $\left.)\right)$. Proof: $\operatorname{dom} \tau \subseteq \operatorname{dom}$ $\left(\tau\right.$ with-replacement $\left(\xi, x_{1}\right.$-term $\left.)\right) \cdot \operatorname{dom}\left(\tau\right.$ with-replacement $\left(\xi, x_{1}\right.$-term $\left.)\right) \subseteq$ $\operatorname{dom} \tau$ by [17, (29)], [20, (3)].

(137) Let us consider trees $\tau, \tau_{1}$ and an element $\xi$ of $\tau$.

Then $\left(\tau\right.$ with-replacement $\left.\left(\xi, \tau_{1}\right)\right)\left\lceil\xi=\tau_{1}\right.$. The theorem is a consequence of $(1)$.

(138) Let us consider decorated trees $\tau, \tau_{1}$ and a node $\xi$ of $\tau$.

Then $\left(\tau\right.$ with-replacement $\left.\left(\xi, \tau_{1}\right)\right)\left\lceil\xi=\tau_{1}\right.$. The theorem is a consequence of (137).

Let us consider a node $\xi$ of $\tau$. Now we state the propositions:

(139) If $\tau_{1}=\tau \mid \xi$, then $h(\tau) \mid \xi=h\left(\tau_{1}\right)$. Proof: Define $\mathcal{P}$ [element of $\left.\mathfrak{F}_{\Sigma}(X)\right] \equiv$ for every node $\xi$ of $\$_{1}$ for every $\tau_{1}$ such that $\tau_{1}=\$_{1} \mid \xi$ holds $h\left(\$_{1}\right)\left\lceil\xi=h\left(\tau_{1}\right)\right.$ and $\xi \in \operatorname{dom}\left(h\left(\$_{1}\right)\right) . \mathcal{P}[x$-term] by [17, (29)], [20, (3)], [21, (1)], [17, (22)]. For every $o$ and $p$ such that for every $\tau$ such that $\tau \in \operatorname{rng} p$ holds $\mathcal{P}[\tau]$ holds $\mathcal{P}[o$-term $p]$ by [20, (11)], [21, (1)], [17, (22)], [21, (3)]. $\mathcal{P}[\tau]$ from TermInd.

(140) If $\tau(\xi)=\langle x, \sigma\rangle$, then $\tau\lceil\xi=x$-term. The theorem is a consequence of (36).

Now we state the propositions:

(141) Let us consider trees $\tau, \tau_{1}$ and elements $\xi, \nu$ of $\tau$. Suppose

(i) $\xi \nsubseteq \nu$, and

(ii) $\nu \nsubseteq \xi$.

Then $\left(\tau\right.$ with-replacement $\left.\left(\xi, \tau_{1}\right)\right)\lceil\nu=\tau\lceil\nu$. The theorem is a consequence of $(2)$ and (5).

(142) Let us consider decorated trees $\tau, \tau_{1}$ and nodes $\xi, \nu$ of $\tau$. Suppose

(i) $\xi \nsubseteq \nu$, and

(ii) $\nu \nsubseteq \xi$.

Then $\left(\tau\right.$ with-replacement $\left.\left(\xi, \tau_{1}\right)\right)\lceil\nu=\tau\lceil\nu$. The theorem is a consequence of (141) and (5).

(143) If $\tau \subseteq \tau_{1}$, then $\tau=\tau_{1}$. Proof: Define $\mathcal{P}$ [element of $\left.\mathfrak{F}_{\Sigma}(X)\right] \equiv$ for every $\tau_{1}$ such that $\$_{1} \subseteq \tau_{1}$ holds $\$_{1}=\tau_{1} . \mathcal{P}[x$-term] by [17, (22)], [30, (2)], [20, (3)], (36). For every $o$ and $p$ such that for every $\tau$ such that $\tau \in \operatorname{rng} p$ holds $\mathcal{P}[\tau]$ holds $\mathcal{P}[o$-term $p]$ by [17, (22)], [30, (2)], (36), [20, (3)]. $\mathcal{P}[\tau]$ from TermInd.

(144) Let us consider an endomorphism $h$ of $\mathfrak{F}_{\Sigma}(X)$. Then

(i) $\operatorname{dom} \tau \subseteq \operatorname{dom}(h(\tau))$, and 
(ii) for every $I$ such that $I=\{\xi$, where $\xi$ is an element of dom $\tau$ : there exists $\sigma$ and there exists $x$ such that $\tau(\xi)=\langle x, \sigma\rangle\}$ holds $\tau\lceil(\operatorname{dom} \tau \backslash$ $I)=h(\tau)\lceil(\operatorname{dom} \tau \backslash I)$.

Proof: Define $\mathcal{P}$ [element of $\left.\mathfrak{F}_{\Sigma}(X)\right] \equiv \operatorname{dom} \$_{1} \subseteq \operatorname{dom}\left(h\left(\$_{1}\right)\right)$ and for every $I$ such that $I=\left\{\xi\right.$, where $\xi$ is an element of dom $\$_{1}$ : there exists $\sigma$ and there exists $x$ such that $\left.\$_{1}(\xi)=\langle x, \sigma\rangle\right\}$ holds $\$_{1}\left\lceil\left(\operatorname{dom} \$_{1} \backslash I\right)=\right.$ $h\left(\$_{1}\right)\left\lceil\left(\operatorname{dom} \$_{1} \backslash I\right) . \mathcal{P}[x\right.$-term] by [17, (22)], [20, (3)], [17, (29)]. For every $o$ and $p$ such that for every $\tau$ such that $\tau \in \operatorname{rng} p$ holds $\mathcal{P}[\tau]$ holds $\mathcal{P}[o$-term $p]$ by (34), [10, (13)], 20, (11)], [17, (22)]. $\mathcal{P}[\tau]$ from TermInd.

(145) Suppose $I=\{\xi$, where $\xi$ is an element of $\operatorname{dom} \tau$ : there exists $\sigma$ and there exists $x$ such that $\tau(\xi)=\langle x, \sigma\rangle\}$. Let us consider a node $\xi$ of $h(\tau)$. Then

(i) $\xi \in \operatorname{dom} \tau \backslash I$, or

(ii) there exists an element $\nu$ of $\operatorname{dom} \tau$ such that $\nu \in I$ and there exists a node $\mu$ of $h(\tau)\left\lceil\nu\right.$ such that $\xi=\nu^{\frown} \mu$.

Proof: Define $\mathcal{P}\left[\right.$ element of $\left.\mathfrak{F}_{\Sigma}(X)\right] \equiv$ for every $I$ such that $I=\{\xi$, where $\xi$ is an element of $\operatorname{dom} \$_{1}$ : there exists $\sigma$ and there exists $x$ such that $\left.\$_{1}(\xi)=\langle x, \sigma\rangle\right\}$ for every node $\xi$ of $h\left(\$_{1}\right), \xi \in \operatorname{dom} \$_{1} \backslash I$ or there exists an element $\nu$ of $\operatorname{dom} \$_{1}$ such that $\nu \in I$ and there exists a node $\mu$ of $h\left(\$_{1}\right)\left\lceil\nu\right.$ such that $\xi=\nu^{\frown} \mu . \mathcal{P}[x$-term] by [17, (22)], [20, (3)], [21, (1)]. For every $o$ and $p$ such that for every $\tau$ such that $\tau \in \operatorname{rng} p$ holds $\mathcal{P}[\tau]$ holds $\mathcal{P}[o$-term $p]$ by (34), [10, (13)], [20, (11)], [17, (22)]. $\mathcal{P}[\tau]$ from TermInd.

(146) Let us consider an endomorphism $h$ of $\mathfrak{F}_{\Sigma}(Y)$ a one-to-one finite sequence $g$ of elements of dom $v$. Suppose

(i) $\operatorname{rng} g=\{\xi$, where $\xi$ is an element of $\operatorname{dom} v$ : there exists $\sigma$ and there exists $y$ such that $v(\xi)=\langle y, \sigma\rangle\}$, and

(ii) $\operatorname{dom} v \subseteq \operatorname{dom} v_{1}$, and

(iii) $v \uparrow(\operatorname{dom} v \backslash \operatorname{rng} g)=v_{1} \uparrow(\operatorname{dom} v \backslash \operatorname{rng} g)$, and

(iv) for every $i$ such that $i \in \operatorname{dom} g$ holds $h(v)\left\lceil\left(g_{i}\right.\right.$ qua node of $\left.v\right)=$ $v_{1} \uparrow\left(g_{i}\right.$ qua node of $\left.v\right)$.

Then $h(v)=v_{1}$. Proof: $h(v)\left\lceil(\operatorname{dom} v \backslash \operatorname{rng} g)=v_{1}\lceil(\operatorname{dom} v \backslash \operatorname{rng} g) . h(v) \subseteq\right.$ $v_{1}$ by [27, (1)], (145), [27, (49)], (144).

(147) Let us consider an endomorphism $h$ of $\mathfrak{F}_{\Sigma}(Y)$ and a vf-sequence $f$ of $v$. Suppose $f \neq \emptyset$. Then there exists a non empty finite sequence $\mathcal{B}$ of elements of the carrier of $\Sigma$ and there exists a $\mathcal{B}$-sorting finite sequence $V_{1}$ of elements of $\bigcup Y$ such that $\operatorname{dom} \mathcal{B}=\operatorname{dom} f$ and $\mathcal{B}=\operatorname{pr} 2(f)$ and $V_{1}=\operatorname{pr} 1(f)$ and there exists a $\mathcal{B}$-sorting finite sequence $D$ of elements 
of $\mathfrak{F}_{\Sigma}(Y)$ and there exists a $V_{1}$-omitting $D$-omitting $\mathcal{B}$-sorting finite sequence $V_{2}$ of elements of $\bigcup Y$ such that for every element $i$ of $\operatorname{dom} \mathcal{B}$, $D(i)=h\left(V_{1}(i)\right.$-term $)$ and there exists a $V_{2}$-context sequence finite sequence $F$ of elements of $\mathfrak{F}_{\Sigma}(Y)$ such that $F$ is $\left(V_{1}, V_{2}, D\right)$-consequent context sequence and $F(1(\in \operatorname{dom} \mathcal{B}))\left[V_{1}(1(\in \operatorname{dom} \mathcal{B}))\right.$-term $]=v$ and $h(v)=F((\operatorname{len} \mathcal{B})(\in \operatorname{dom} \mathcal{B}))[D((\operatorname{len} \mathcal{B})(\in \operatorname{dom} \mathcal{B}))]$. Proof: Reconsider $\mathcal{B}=\operatorname{pr} 2(f)$ as a non empty finite sequence of elements of the carrier of $\Sigma$. Consider $g$ being a one-to-one finite sequence such that $\operatorname{rng} g=\{\xi$, where $\xi$ is an element of $\operatorname{dom} v$ : there exists $\sigma$ and there exists $y$ such that $v(\xi)=\langle y, \sigma\rangle\}$ and $\operatorname{dom} f=\operatorname{dom} g$ and for every $i$ such that $i \in \operatorname{dom} f$ holds $f(i)=v(g(i)) \operatorname{rng} g \subseteq \operatorname{dom} v$. Reconsider $V_{1}=\operatorname{pr} 1(f)$ as a $\mathcal{B}$ sorting finite sequence of elements of $\bigcup Y$. Define $\mathcal{F}($ element of dom $\mathcal{B})=$ $h\left(V_{1}\left(\$_{1}\right)\right.$-term). Consider $D$ being a non empty finite sequence such that $\operatorname{dom} D=\operatorname{dom} \mathcal{B}$ and for every element $i$ of $\operatorname{dom} \mathcal{B}, D(i)=\mathcal{F}(i)$ from FinSeqLambda. $D$ is a finite sequence of elements of $\mathfrak{F}_{\Sigma}(Y)$. D is $\mathcal{B}$-sorting. Set $V_{2}=$ the one-to-one $V_{1}$-omitting $D$-omitting $\mathcal{B}$-sorting finite sequence of elements of $\bigcup Y$. Define $\mathcal{H}$ (element of dom $\mathcal{B}$, decorated tree $)=\left(\$_{2}\right.$ withreplacement $\left(\left(\left(g_{\$_{1}}\right.\right.\right.$ qua element of $\left.\operatorname{dom} v\right)$ qua finite sequence of elements of $\left.\left.\mathbb{N}), D\left(\$_{1}\right)\right)\right)$ with-replacement $\left(\left(\left(g_{\$_{1}+1}\right.\right.\right.$ qua element of $\left.\operatorname{dom} v\right)$ qua finite sequence of elements of $\mathbb{N})$, the root tree of $\left.\left\langle V_{2}\left(\$_{1}+1\right), \mathcal{B}\left(\$_{1}+1\right)\right\rangle\right)$. Consider $F$ being a non empty decorated tree yielding finite sequence such that $\operatorname{dom} F=\operatorname{dom} \mathcal{B}$ and $F(1)=v$ with-replacement $\left(\left(\left(g_{1}\right.\right.\right.$ qua element of $\operatorname{dom} v$ ) qua finite sequence of elements of $\mathbb{N})$, the root tree of $\left\langle V_{2}(1)\right.$, $\mathcal{B}(1)\rangle)$ and for every elements $i, j$ of $\operatorname{dom} \mathcal{B}$ such that $j=i+1$ for every decorated tree $d$ such that $d=F(i)$ holds $F(j)=\mathcal{H}(i, d)$ from FinSeqRec2Lambda. $\operatorname{rng} F \subseteq \bigcup$ (the sorts of $\left.\mathfrak{F}_{\Sigma}(Y)\right)$ by (131), [22, (87)], [20, (3)], (133). Define $\mathcal{Q}$ [natural number] $\equiv$ for every element $b$ of $\operatorname{dom} \mathcal{B}$ such that $\$_{1}=b$ holds $F(b)$ is a context of $V_{2}(b)$ and $\operatorname{dom} v \subseteq \operatorname{dom}(F(b))$ and $F(b)\left(g_{b}\right)=\left\langle V_{2}(b), \mathcal{B}(b)\right\rangle$ and for every element $b_{1}$ of dom $\mathcal{B}$ such that $b_{1}>b$ holds $F_{b}$ is $\left(V_{2}\left(b_{1}\right)\right)$-omitting and $F(b)\left(g_{b_{1}}\right)=\left\langle V_{1}\left(b_{1}\right), \mathcal{B}\left(b_{1}\right)\right\rangle . \mathcal{Q}[1]$ by [27, (102)], (134), (135), [22, (87)]. For every $i$ such that $1 \leqslant i$ and $\mathcal{Q}[i]$ holds $\mathcal{Q}[i+1]$ by [52, (25)], [13, (13)], [27, (102)], (132). For every $i$ such that $i \geqslant 1$ holds $\mathcal{Q}[i]$ from [13, Sch. 8]. $F$ is $V_{2}$-context sequence by [52, (25)]. $F$ is $\left(V_{1}, V_{2}, D\right)$-consequent context sequence by [52, (25)], [13, (12), (13)], (132). Set $b=1(\in \operatorname{dom} \mathcal{B})$. Reconsider $\nu=g_{b}, \xi=g_{\text {len } \mathcal{B}}$ as a node of $v$. Consider $\mu$ being a node of $v$ such that $\nu=\mu$ and there exists $\sigma$ and there exists $y$ such that $v(\mu)=\langle y, \sigma\rangle \cdot \operatorname{dom}(F(b))=\operatorname{dom} v$. Reconsider $\tau=V_{1}(b)$-term as an element of $\mathfrak{F}_{\Sigma}(Y)$. Consider $\mu$ being a finite sequence of elements of $\mathbb{N}$ such that $\mu \in \operatorname{dom}\left(V_{2}(b)\right.$-term) and $\nu=\nu^{\frown} \mu$ and $F(b)(\nu)=V_{2}(b)-\operatorname{term}(\mu) . F(b)[\tau]=F(b)$ with-replacement $(\nu, \tau)$. Define $\Sigma$ [natural number] $\equiv$ for every elements $b, b_{1}$ of $\operatorname{dom} \mathcal{B}$ such that $\$_{1}=b$ and $b_{1} \leqslant b$ holds $(F(b)[D(b)]) \uparrow\left(g_{b_{1}}\right.$ qua node of $\left.v\right)=h(v) \uparrow\left(g_{b_{1}}\right.$ qua node of 
$v)$ and $(F(b)[D(b)])\lceil(\operatorname{dom} v \backslash \operatorname{rng} g)=v \uparrow(\operatorname{dom} v \backslash \operatorname{rng} g) . \Sigma[1]$ by [52, (25)], (132), (138), (140). For every $i$ such that $i \geqslant 1$ and $\Sigma[i]$ holds $\Sigma[i+1]$ by [52, (25)], [13, (13)], (132), (135). Set $b=(\operatorname{len} \mathcal{B})(\in \operatorname{dom} \mathcal{B})$. Set $v_{1}=$ $F(b)[D(b)]$. For every $i$ such that $i \geqslant 1$ holds $\Sigma[i]$ from [13, Sch. 8]. $v_{1}=$ $F(b)$ with-replacement $\left(\left(g_{b}\right.\right.$ qua node of $\left.\left.v\right), D(b)\right) \cdot \operatorname{dom}(F(b)) \subseteq \operatorname{dom} v_{1}$.

\section{REFERENCES}

[1] Grzegorz Bancerek. Towards the construction of a model of Mizar concepts. Formalized Mathematics, 16(2):207-230, 2008. doi 10.2478/v10037-008-0027-x

[2] Grzegorz Bancerek. Cardinal numbers Formalized Mathematics, 1(2):377-382, 1990.

[3] Grzegorz Bancerek. Cardinal arithmetics. Formalized Mathematics, 1(3):543-547, 1990.

[4] Grzegorz Bancerek. König's theorem. Formalized Mathematics, 1(3):589-593, 1990.

[5] Grzegorz Bancerek. On powers of cardinals. Formalized Mathematics, 3(1):89-93, 1992.

[6] Grzegorz Bancerek. Algebra of morphisms Formalized Mathematics, 6(2):303-310, 1997.

[7] Grzegorz Bancerek. Tarski's classes and ranks. Formalized Mathematics, 1(3):563-567, 1990.

[8] Grzegorz Bancerek. Cartesian product of functions. Formalized Mathematics, 2(4):547$552,1991$.

[9] Grzegorz Bancerek. Institution of many sorted algebras. Part I: Signature reduct of an algebra. Formalized Mathematıcs, 6(2):279-287, 1997.

[10] Grzegorz Bancerek. Free term algebras. Formalized Mathematics, 20(3):239-256, 2012. doi:10.2478/v10037-012-0029-6

[11] Grzegorz Bancerek. Terms over many sorted universal algebra. Formalized Mathematics, 5(2):191-198, 1996.

[12] Grzegorz Bancerek. Translations, endomorphisms, and stable equational theories. Formalized Mathematics, 5(4):553-564, 1996.

[13] Grzegorz Bancerek. The fundamental properties of natural numbers Formalized Mathematics, 1(1):41-46, 1990.

[14] Grzegorz Bancerek. The ordinal numbers, Formalized Mathematics, 1(1):91-96, 1990.

[15] Grzegorz Bancerek. Veblen hierarchy. Formalized Mathematics, 19(2):83-92, 2011. doi $10.2478 / v 10037-011-0014-5$

[16] Grzegorz Bancerek. Reduction relations, Formalized Mathematics, 5(4):469-478, 1996.

[17] Grzegorz Bancerek. Introduction to trees Formalized Mathematics, 1(2):421-427, 1990.

[18] Grzegorz Bancerek. König's lemma Formalized Mathematics, 2(3):397-402, 1991.

[19] Grzegorz Bancerek. Sets and functions of trees and joining operations of trees Formalized Mathematics, 3(2):195-204, 1992.

[20] Grzegorz Bancerek. Joining of decorated trees Formalized Mathematics, 4(1):77-82, 1993.

[21] Grzegorz Bancerek. Subtrees. Formalized Mathematics, 5(2):185-190, 1996.

[22] Grzegorz Bancerek and Krzysztof Hryniewiecki. Segments of natural numbers and finite sequences. Formalized Mathematics, 1(1):107-114, 1990.

[23] Grzegorz Bancerek and Artur Korniłowicz. Yet another construction of free algebra Formalized Mathematics, 9(4):779-785, 2001.

[24] Grzegorz Bancerek and Piotr Rudnicki. On defining functions on trees Formalized $\mathrm{Ma}$ thematics, 4(1):91-101, 1993.

[25] Grzegorz Bancerek and Andrzej Trybulec. Miscellaneous facts about functions Formalized Mathematics, 5(4):485-492, 1996.

[26] Czesław Byliński. Finite sequences and tuples of elements of a non-empty sets. Formalized Mathematics, 1(3):529-536, 1990.

[27] Czesław Byliński. Functions and their basic properties. Formalized Mathematics, 1(1): 55-65, 1990.

[28] Czesław Byliński. Functions from a set to a set Formalized Mathematics, 1(1):153-164, 1990. 
[29] Czesław Byliński. The modification of a function by a function and the iteration of the composition of a function Formalized Mathematıcs, 1(3):521-527, 1990.

[30] Czesław Byliński. Graphs of functions Formalized Mathematics, 1(1):169-173, 1990.

[31] Czesław Byliński. Partial functions. Formalized Mathematics, 1(2):357-367, 1990.

[32] Czesław Byliński. Some basic properties of sets. Formalized Mathematics, 1(1):47-53, 1990.

[33] Agata Darmochwał. Finite sets Formalized Mathematics, 1(1):165-167, 1990.

[34] Andrzej Kondracki. Basic properties of rational numbers Formalized Mathematics, 1(5): 841-845, 1990.

[35] Andrzej Kondracki. The Chinese Remainder Theorem Formalized Mathematics, 6(4): 573-577, 1997.

[36] Małgorzata Korolkiewicz. Homomorphisms of many sorted algebras Formalized Mathematics, 5(1):61-65, 1996.

[37] Małgorzata Korolkiewicz. Many sorted quotient algebra. Formalized Mathematics, 5(1): 79-84, 1996.

[38] Jarosław Kotowicz. Convergent real sequences. Upper and lower bound of sets of real numbers. Formalized Mathematıcs, 1(3):477-481, 1990.

[39] Yatsuka Nakamura. Determinant of some matrices of field elements. Formalized Mathematics, 14(1):1-5, 2006. doi 10.2478/v10037-006-0001-4

[40] Hiroyuki Okazaki, Yuichi Futa, and Yasunari Shidama. Constructing binary Huffman tree. Formalized Mathematics, 21(2):133-143, 2013. doi:10.2478/forma-2013-0015

[41] Beata Perkowska. Free many sorted universal algebra Formalized Mathematics, 5(1) 67-74, 1996.

[42] Karol Pak. Abstract simplicial complexes. Formalized Mathematics, 18(1):95-106, 2010. doi $10.2478 / v 10037-010-0013-y$

[43] Andrzej Trybulec. Domains and their Cartesian products. Formalized Mathematics, 1(1): 115-122, 1990.

[44] Andrzej Trybulec. Function domains and Frænkel operator. Formalized Mathematics, 1 (3):495-500, 1990 .

[45] Andrzej Trybulec. Binary operations applied to functions. Formalized Mathematics, 1 (2):329-334, 1990.

[46] Andrzej Trybulec. Tuples, projections and Cartesian products. Formalized Mathematics, 1(1):97-105, 1990.

[47] Andrzej Trybulec. On the sets inhabited by numbers Formalized Mathematics, 11(4): 341-347, 2003.

[48] Andrzej Trybulec. A scheme for extensions of homomorphisms of many sorted algebras Formalized Mathematıcs, 5(2):205-209, 1996.

[49] Andrzej Trybulec. Many sorted algebras. Formalized Mathematics, 5(1):37-42, 1996.

[50] Andrzej Trybulec. Many sorted sets Formalized Mathematics, 4(1):15-22, 1993.

[51] Michał J. Trybulec. Integers. Formalized Mathematics, 1(3):501-505, 1990.

[52] Wojciech A. Trybulec. Non-contiguous substrings and one-to-one finite sequences Formalized Mathematics, 1(3):569-573, 1990.

[53] Wojciech A. Trybulec. Pigeon hole principle Formalized Mathematics, 1(3):575-579, 1990.

[54] Zinaida Trybulec. Properties of subsets. Formalized Mathematics, 1(1):67-71, 1990.

[55] Edmund Woronowicz. Relations and their basic properties Formalized Mathematics, 1 (1):73-83, 1990.

[56] Edmund Woronowicz. Relations defined on sets Formalized Mathematics, 1(1):181-186, 1990. 